\title{
Hedgehog signaling pathway affects the sensitivity of hepatoma cells to drug therapy through the $A B C C 1$ transporter
}

\author{
Jia Ding ${ }^{1}$, Xiao-Tian Zhou ${ }^{2}$, Hao-Yu Zou ${ }^{2}$ and Jian Wu ${ }^{2}$
}

The poor response to drug therapy often seen in hepatocellular carcinoma requires insight into the molecular interplay

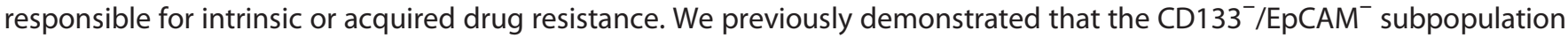
of the Huh-7 hepatoma cell line features aberrant activation of the hedgehog signaling ( $\mathrm{Hh}$ ) pathway and chemoresistance. The prevailing hypothesis of the present study is that hedgehog signaling may govern expression of ATP-binding cassette $(\mathrm{ABC})$ transporters, which are responsible for drug resistance in the $\mathrm{CD}^{133^{-}} / \mathrm{EpCAM}^{-}$subpopulation. Our aim is to reveal the molecular interplay in the mediation of drug resistance with a newly established Huh-7 subpopulation featuring high Hh signaling activity and drug resistance. In this study, chemoresistance was determined in a newly established Huh-7-DN subpopulation featuring the $\mathrm{CD}_{133^{-}} / \mathrm{EpCAM}^{-}$surface marker profile, aberrant expression of Hh pathway, and epithelial-mesenchymal transition (EMT). Expression of $A B C$ transporter proteins $(A B C B 1, A B C C 1$, and $A B C G 2)$ and $\mathrm{Hh}$ transcription factor Gli-1/2 was evaluated with and without Hh signaling antagonists LDE225 or itraconazole. We found that hedgehog signaling activity as determined by transfection with a Gli-Lux reporter cassette

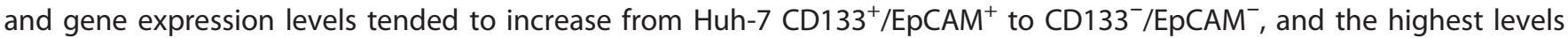
were found in Huh-7-DN cells. The Huh-7-DN subpopulation exhibited characteristics of EMT as evidenced by increased expression of vimentin and loss of E-cadherin. Sorafenib significantly inhibited the viability of all subpopulations except the Huh-7-DN subpopulation. Compared with other sorafenib-sensitive subpopulations, the Huh-7-DN subpopulation showed enhanced expression of $\mathrm{Hh}$ transcription factor Gli-2 and ABCC1 transporter protein. Silencing Gli-2 by lentivirus harboring shRNA against Gli-2 or LDE225 significantly suppressed expression of Gli-2 and ABCC1 genes in Huh-7-DN subpopulation. In conclusion, aberrant hedgehog signaling activation is linked to poor differentiation, epithelialmesenchymal transition, and chemoresistance in the Huh-7-DN subpopulation. Hedgehog signaling transcription factor Gli-2 appears to be the primary regulator for drug sensitivity of hepatoma through the ABCC1 transporter.

Laboratory Investigation (2017) 97, 819-832; doi:10.1038/labinvest.2017.34; published online 17 April 2017

As the third most common death of malignancy, more than 700000 new cases of hepatocellular carcinoma (HCC) occur worldwide every year. ${ }^{1}$ Despite the steadily increasing incidence, only $25 \%$ of patients benefit from surgical intervention. ${ }^{2}$ The advanced stage at the time of diagnosis, aggressive behavior of HCC progression, the high recurrence after resection $(60-80 \%$ in 5 years), and ablation $(40-70 \%)$ are major causes of a very low 5-year survival rate. ${ }^{3}$ Limited adjuvant therapies are available for advanced HCC patients. ${ }^{4,5}$ Sorafenib, a Raf-1 kinase inhibitor, was reported to extend patient survival for nearly 3 months. ${ }^{6}$ However, a recent multicenter, double-blinded trial concluded that sorafenib did not extend recurrence-free survival, but increased adverse effects of the treatment, ${ }^{3}$ and the findings are consistent with a growing list of negative reports from primary or adjuvant use after resection, ablation, or transarterial chemoembolization. $^{7}$

Primary or acquired multidrug resistance (MDR) is one of the reasons leading to HCC treatment failure and progression. ${ }^{8}$ It is known that MDR increases drug efflux by

\footnotetext{
'Department of Gastroenterology, Huashan Hospital, Fudan University, Shanghai, China and ${ }^{2}$ Department of Medical Microbiology, Key Laboratory of Molecular Virology, School of Basic Medical Sciences, Fudan University Shanghai Medical College, Shanghai, China

Correspondence: Professor J Wu, MD, PhD, Department of Medical Microbiology, Key Laboratory of Molecular Virology, School of Basic Medical Sciences, Fudan University Shanghai Medical College, 138 Yixue Yuan Road, PO Box 228, Shanghai 200032, China.

E-mail: jian.wu@fudan.edu.cn

The abstract of this work was presented in the 66th Annual Meeting of the American Association for the Study of Liver Disease (AASLD), 11-15 November 2016, Boston, MA, USA.

Received 7 September 2016; revised 18 January 2017; accepted 7 February 2017
} 
adenosine triphosphate binding cassette $(\mathrm{ABC})$ transporters, resulting in a decrease in intracellular concentrations of chemotherapeutic agents. ABCB1 (MDR-1, P-glycoprotein), ABCC1 (multidrug resistance-associated protein 1, MRP1), and ABCG2 (breast cancer-resistance protein, BCRP) are the best-characterized $\mathrm{ABC}$ transporters that contribute to chemoresistance. ${ }^{9}$ However, it is not yet understood how $\mathrm{ABC}$ transporter proteins affect the development of intrinsic and acquired resistance to sorafenib in HCC.

We previously demonstrated that hepatoma cell lines could be separated into subpopulations according to the expression profile of cell surface markers CD133 and EpCAM. The $\mathrm{CD} 133^{-} / \mathrm{EpCAM}^{-}$subpopulation is more resistant to cisplatin, doxorubicin, and sorafenib in comparison with the $\mathrm{CD} 133^{+} / \mathrm{EpCAM}^{+}$subpopulation. ${ }^{10}$ Moreover, our data suggest that the chemoresistance is correlated with aberrant activation of the hedgehog ( $\mathrm{Hh}$ ) signaling pathway and occurrence of epithelial-mesenchymal transition (EMT). ${ }^{10}$ Hedgehog signaling is quiescent in adult tissues. Overexpression of $\mathrm{Hh}$ pathway molecules, such as sonic $\mathrm{Hh}$, PTCH1, SMO, and Gli-1/2, has been reported in $60-70 \%$ of HCC specimens, ${ }^{11,12}$ and dysregulated expression of $\mathrm{Hh}$ molecules is one of disordered signaling mechanisms crucial for hepatic carcinogenesis ${ }^{13}$ and highly metastatic behavior of HCC. ${ }^{14}$ Hh signaling inhibitors, such as vismodegib (GDC-0449) and sonidegib (LDE225), are approved for the treatment of advanced basal cell carcinoma; ${ }^{15}$ and sonidegib is in phase I-II clinical trials for the treatment of a variety of malignancies, including esophageal, pancreatic, ovarian cancers, and hepatocellular carcinoma. ${ }^{16}$ However, whether abnormal hedgehog signaling has a critical role in modulating drug resistance through $\mathrm{ABC}$ transporter molecules has not been thoroughly investigated. Therefore, we tested the hypothesis that hedgehog signaling may govern expression of $\mathrm{ABC}$ transporter proteins, which are responsible for chemoresistance in hepatoma cells.

In the present study we established a unique Huh-7 subpopulation, which features higher hedgehog signaling activity with EMT characteristics and drug resistance. We investigated how drug resistance is developed and the underlying controlling mechanisms within this specific subpopulation.

\section{MATERIALS AND METHODS \\ Cell Culture and Reagents}

Hepatoma cell lines, Huh-7 and Hep3B, were obtained from the American Type Culture Collection (Manassas, VA, USA). The Hep3B cells were incubated in minimum essential medium (MEM, Gibco, Grand Island, NY, USA), and Huh7 cells were incubated in Dulbecco's modified essential medium (DMEM, Gibco), supplemented with 10\% heatinactivated fetal bovine serum (FBS, Gibco) and 1\% (v/v) penicillin-streptomycin.

\section{Fluorescence Activation Cell Sorting Enrichment and Transwell Matrigel Invasion Assay for Subpopulation Selection}

$\mathrm{CD} 133^{+} / \mathrm{EpCAM}^{+}$and $\mathrm{CD} 133^{-} / \mathrm{EpCAM}^{-}$subpopulations were enriched from Huh-7 cells by fluorescence activation cell sorting (FACS) using allophycocyanin (APC)-conjugated monoclonal antibodies against human CD133 and fluorescein isothiocyanate (FITC)-conjugated monoclonal antibodies against human EpCAM as we previously described. ${ }^{10}$ Briefly, the Huh-7 cells were detached by $0.05 \%$ Trypsin-EDTA (Gibco) and incubated with corresponding antibodies (CD133/EpCAM) for $45 \mathrm{~min}$. After washing, the cells were suspended in DMEM containing 10\% FBS until sorting. All the cells were sorted in a high-speed MoFlo XDP Cell Sorter (Beckman, Indianapolis, IN, USA). The Huh-7-DN subpopulation, which stably retained negative CD133/EpCAM expression profile was isolated by FACS enrichment plus culture selection from $\mathrm{CD}_{13}{ }^{-} / \mathrm{EpCAM}^{-}$subpopulation. The Huh-7-trans subpopulation was isolated using Matrigel invasion transwell selection as we previously described. ${ }^{14}$

\section{Immunofluorescent Staining}

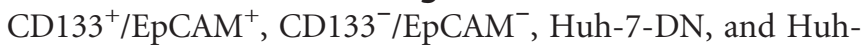
7-trans subpopulations were seeded on coverslips and fixed in $4 \%$ buffered paraformaldehyde. Then cells on coverslips were incubated with primary antibodies against Gli-1, E-cadherin, vimentin, Gli-2, and ABCC1 overnight at $4{ }^{\circ} \mathrm{C}$, and were stained with secondary antibodies (Alexa Fluor 488-conjugated donkey anti-mouse IgG or Alexa Fluor 594/488conjugated donkey anti-rabbit IgG) as we previously described. ${ }^{17}$ All the cells were counter-stained with 4', 6-diamidino-2-phenylindole (DAPI) for nuclear visualization. All electronic images were captured under a Leica TCS SP8 Confocal laser scanning microscope. The sources of all antibodies were listed in the Supplementary Table 1.

\section{Hh Signaling Activity by a Gli-Lux Reporter System}

The Gli-Lux reporter system, in which the firefly luciferase gene is driven by the Gli promoter, was used to determine $\mathrm{Hh}$ signaling activity in different subpopulations. pRL-thymidine kinase (TK) renilla luciferase control reporter (Promega, Madison, WI, USA) was used for transfection normalization. The Gli-Lux reporter system was kindly provided by $\mathrm{Dr}$ Hiroshi Sasaki from the RIKEN Center for Developmental Biology, Kobe, Hyogo, Japan. ${ }^{18}$ FACS-enriched Huh-7

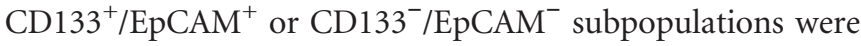
transfected with both Gli-Lux and pRL-TK reporter gene plasmids by Lipofectamine 2000 (Invitrogen, Carlsbad, CA, USA). To determine the inhibitory effect of Hh antagonists, culture medium was replaced with medium containing itraconazole or LDE225 $24 \mathrm{~h}$ after transfection. Firefly and renilla luciferase activity in transfected cells was determined by a dual-luciferase reporter system (Promega). ${ }^{19}$ 


\section{Assays of Cell Viability}

$\mathrm{CD}_{133^{+}} / \mathrm{EpCAM}^{+}, \mathrm{CD} 133^{-} / \mathrm{EpCAM}^{-}$, Huh-7-DN, and Huh7 -trans subpopulations were seeded at $10^{4} /$ well in 96 -well plates and allowed to adhere overnight. The cells were treated with Sorafenib (Selleck, TX, USA), Itraconazole (Biovision, Milpitas, CA, USA) and LDE225 (Cellagen Technology, San Diego, CA, USA) at various concentrations for $24 \mathrm{~h}$. The cell viability was assayed with thiazolyl blue tetrazolium bromide (MTT) assay. ${ }^{20}$ The MTT assay was also used for the determination of IC50 in various cell types in exposure to sorafenib as shown in the Supplementary Information.

\section{RNA Isolation and Quantitative RT-PCR}

Total RNA was extracted from various cells using RNAprep Pure Cell kit (TIANGEN, Beijing, China) according to the manufacturer's instruction. cDNA was synthesized using PrimeScript RT reagent Kit (TAKARA, Dalian, China). Quantitative RT-PCR was performed with Power SYBR Green PCR Master Mix (Applied Biosystems, Foster City, CA, USA) in the Eppendorf AG 22331 RT-PCR system (Eppendorf, Hamburg, German). Primer pair sequences for Gli-1, Gli-2, ABCB1, ABCC1, ABCG2, Twist, Snail, Bcl-2, and $B a x$ are listed in Supplementary Table 2. Relative gene expression was normalized to the housekeeping gene, human glyceraldehyde phosphate dehydrogenase $(G A P D H)$ and expressed as $2^{(-\Delta \Delta \mathrm{CT})}$ as previously described. ${ }^{21,22}$ cDNA generated from human primary hepatocytes was provided by Dr Ping Zhou, Stem Cell Program, UC Davis Medical Center, Sacramento, CA, USA, and used as a control for human gene expression level. Human primary hepatocytes were obtained from the Liver Tissue Procurement and Distribution System (LTPDS), Pittsburgh, PA, USA.

\section{Western Blot Analysis}

Total protein was extracted from different cell subpopulations using RIPA buffer (Ruian BioTechnologies, Shanghai, China). The protein concentration was determined using a bicinchoninic acid (BCA) protein assay (Pierce Biotech, Rockford, USA). Protein lysate $(40 \mu \mathrm{g})$ was loaded and separated by $4-12 \%$ gradient Tris-glycine gels and was transferred to a polyvinylidene fluoride (PVDF) membrane (Millipore, MA, USA) at $80 \mathrm{~V}$ for $1.5 \mathrm{~h}$. Then, membrane of protein extracts was blocked with 5\% skim milk and incubated with monoclonal anti-Gli-1, ABCG2, ABCC1, and $\beta$-actin or polyclonal anti-Gli-2 in Tris-buffered saline containing $0.1 \%$ bovine serum albumin (BSA) overnight at $4{ }^{\circ} \mathrm{C}$. Blotted membranes were incubated with horseradish peroxidaseconjugated secondary antibodies (Cell Signaling Technology, Danvers, MA, USA) for $1 \mathrm{~h}$ at room temperature. Specific protein bands were visualized by the enhanced chemiluminescent reagent (Tanon, Shanghai, China) as previously reported. ${ }^{14}$

\section{Statistical Analysis}

All the experiments were performed three times with a minimum of triplicates. Statistical analysis was performed with SPSS 20.0. The data were expressed as means \pm standard deviation (s.d.), and analyzed by independent-samples $T$-test between two groups or one-way ANOVA for difference between more than two groups, and further by NewmanKeuls or Bonferroni tests for multiple comparisons between given two groups. $P<0.05$ was considered as statistically significant.

\section{RESULTS}

Stable CD133-/EpCAM ${ }^{-}$Expression Profile of Huh-7-DN and Huh-7-Trans Subpopulations

Well-differentiated hepatoma Huh-7 cells were separated into

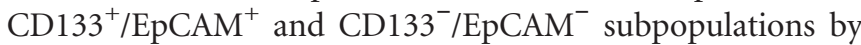
FACS enrichment. As shown in Figure 1a, CD133 ${ }^{+} / \mathrm{EpCAM}^{+}$

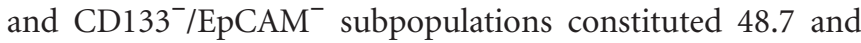
$21.0 \%$ of unsorted Huh-7 cells. Flow cytometric analysis verified that more than $80 \%$ of freshly sorted $\mathrm{CD} 133^{+}$

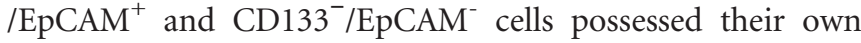
surface marker profile (Figures $1 \mathrm{~b}$ and $\mathrm{c}$ ). However, freshly

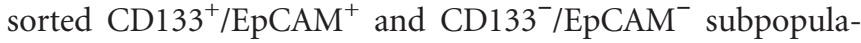
tions gradually lost characteristics of their surface marker profile, and tended to return to an unsorted state after a few passages. To obtain a subpopulation, which stably retained CD133-/EpCAM ${ }^{-}$expression profile, Huh-7-DN and Huh-7trans subpopulations were established by FACS enrichment plus culture selection or Matrigel invasion transwell selection. Both Huh-7-DN and Huh-7-trans subpopulations remained

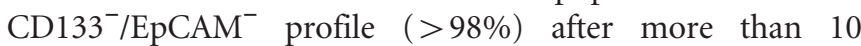
passages (Figures $1 \mathrm{~d}$ and e).

\section{Huh-7-DN Cells were Positive for Hh Transcription Factors Gli-1/2 and EMT Markers}

As shown in Figure 2, Gli-1 and Gli-2 were positive in $\mathrm{CD} 133^{-} / \mathrm{EpCAM}^{-}$subpopulations, especially in Huh-7-DN and Huh-7-trans subpopulations. Staining of EMT markers demonstrated that E-cadherin was only observed in $\mathrm{CD} 133^{+}$ $/ \mathrm{EpCAM}^{+}$subpopulation, whereas other three subpopulations with a negative CD133/EpCAM expression profile exhibited obvious vimentin positivity and loss of E-cadherin expression, indicating that these subpopulations with $\mathrm{CD}_{133^{-}} / \mathrm{EpCAM}^{-}$ expression acquired an EMT status.

\section{Enhanced Hh Signaling Activity in $\mathrm{CD}_{133^{-}} / \mathrm{EpCAM}^{-}$ Subpopulation}

To evaluate the Hh signaling activity, freshly sorted CD $133^{+}$

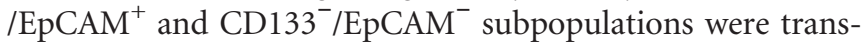
fected with the Gli-Lux firefly reporter cassette, and co-transfected with pRL-TK renilla luciferase reporter control vector for the normalization of transfection efficiency. Two days after transfection, normalized luciferase activity of $\mathrm{CD}^{2} 3^{-} / \mathrm{EpCAM}^{-}$subpopulation was 1.8 -fold higher than $\mathrm{CD} 133^{+} / \mathrm{EpCAM}^{+}$subpopulation $(P<0.01)$. These two 

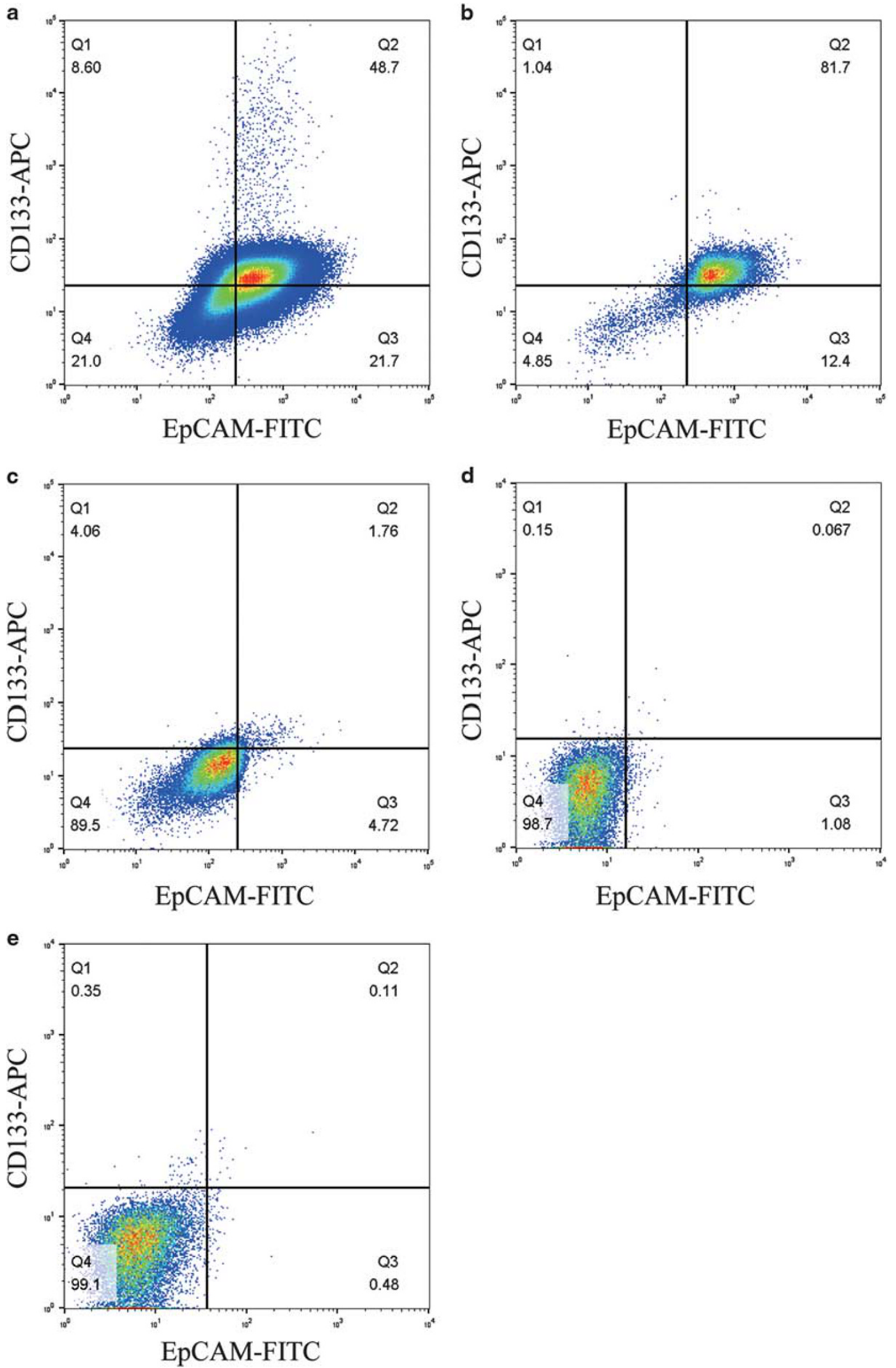

Figure 1 Huh-7-DN and Huh-7-trans subpopulations retained CD133 ${ }^{-} / \mathrm{EpCAM}^{-}$cell surface marker profile over multiple passages. (a) Unsorted Huh-7;

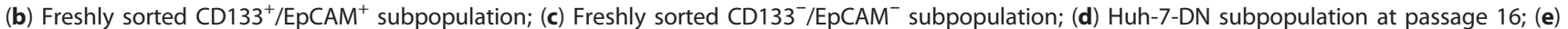
Huh-7-trans subpopulation. 


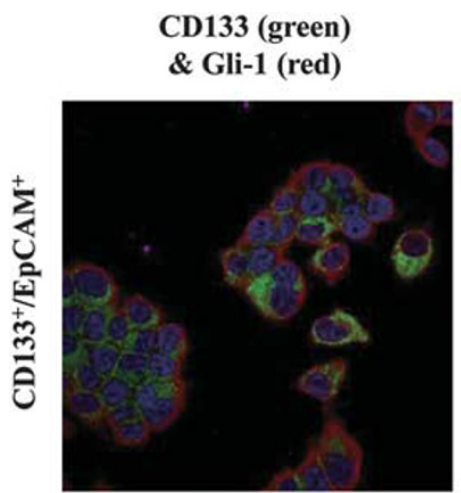

Gli-2 (red)
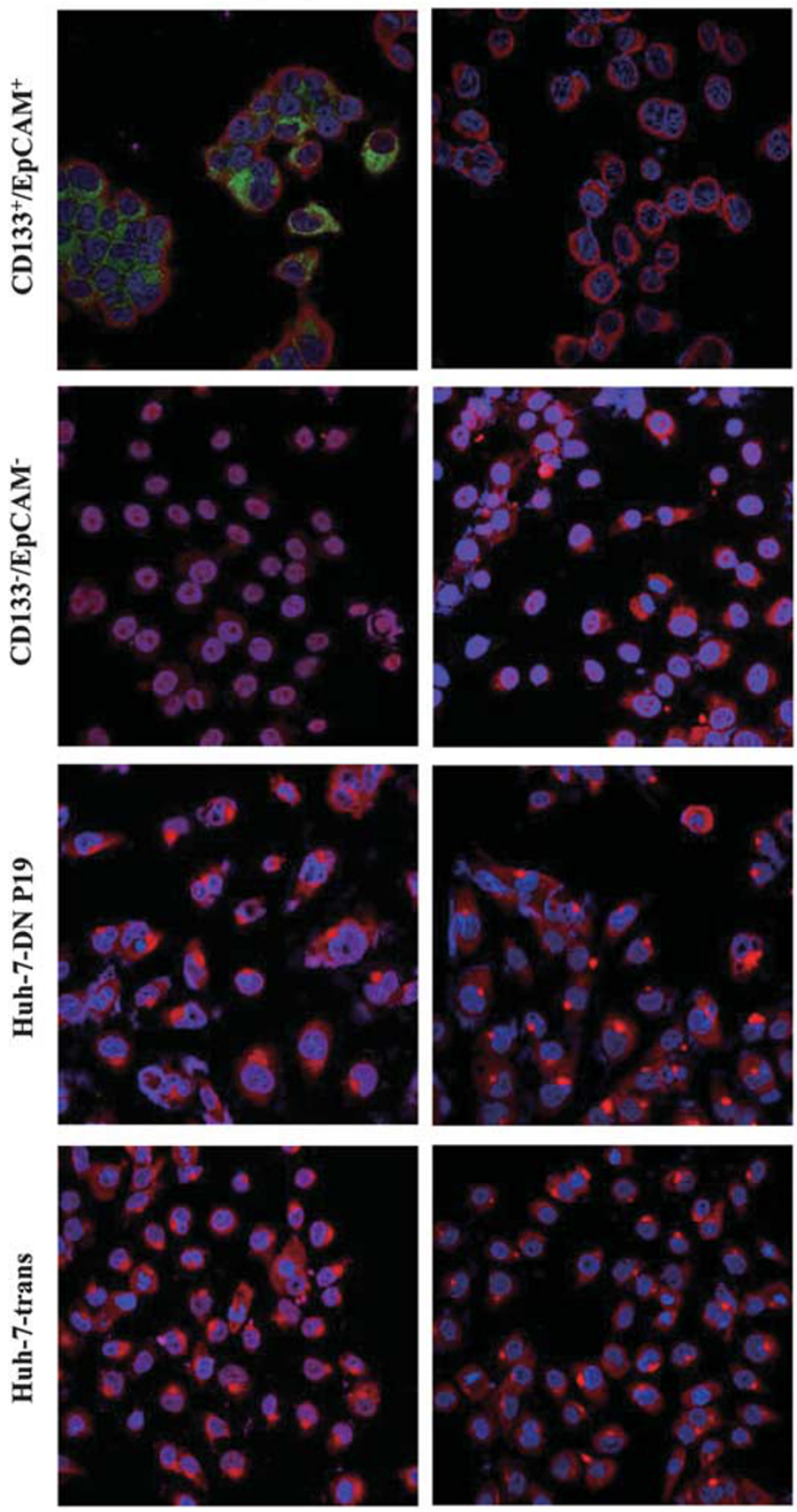
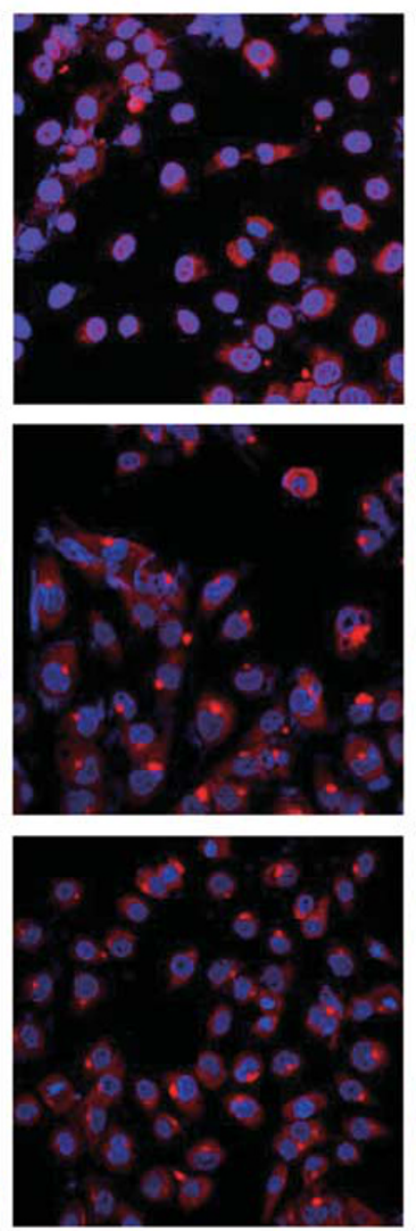

Vimentin (green)
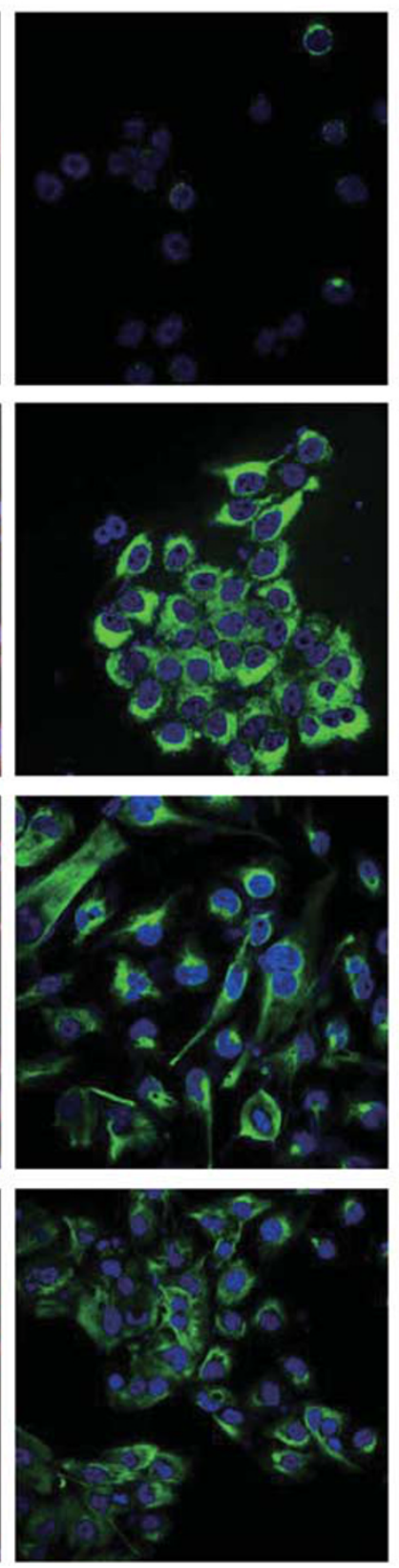

E-cadherin (red)
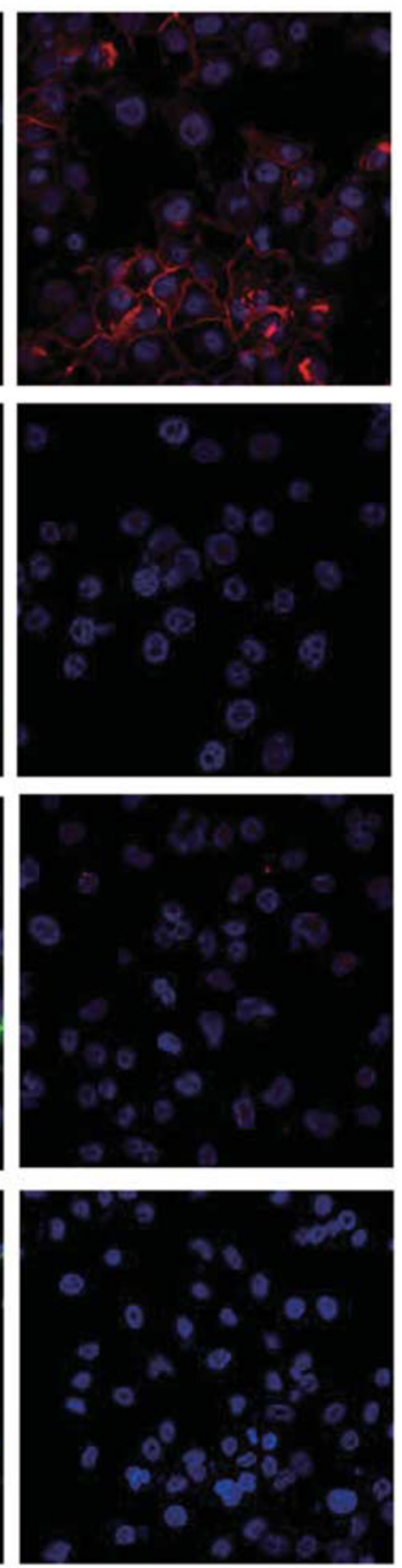

Figure 2 Immunofluorescent staining of Hh signaling transcription factors Gli-1/2 and EMT markers in various Huh-7 subpopulations. CD133 ${ }^{+} / \mathrm{EpCAM}^{+}$,

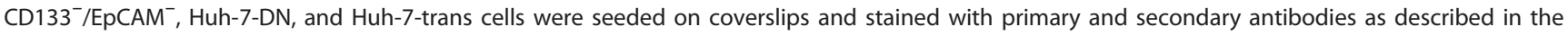
'Materials and Methods' section. All subpopulations were counter-stained with DAPI for nuclear imaging. Magnification: $\times 400$.

subpopulations did not show any significant difference in $\mathrm{Hh}$ signaling activity when transfected with a mutated Gli-Lux reporter cassette (Figure 3a). Moreover, both itraconazole and LDE225, two Hh signaling SMO antagonists, significantly suppressed luciferase activity of $\mathrm{CD}_{133^{-} / \mathrm{EpCAM}^{-} \text {subpopu- }}$ lation (Figures $3 \mathrm{~b}$ and c). Notably, LDE225 was effective at $100 \mathrm{nM}$ in suppressing $\mathrm{Hh}$ signaling activity in this sub-

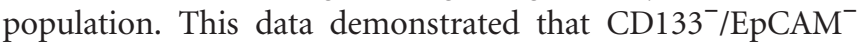
subpopulation displayed higher $\mathrm{Hh}$ signaling activity than
$\mathrm{CD}_{133^{+}} / \mathrm{EpCAM}^{+}$subpopulation, and that specific antagonists targeting the SMO molecule were effective in suppressing $\mathrm{Hh}$ signaling activity in this subpopulation.

\section{Intrinsic Chemoresistance of Huh-7-DN Subpopulation}

To investigate the influence of $\mathrm{Hh}$ signaling activity on drug sensitivity in Huh-7 subpopulations with varying Hh signal-

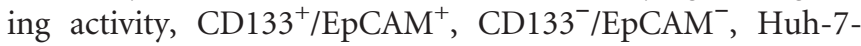
DN, and Huh-7-trans subpopulations were treated with 
a

$$
\begin{aligned}
& \square \text { Huh-7 +/+ } \\
& \text { Huh-7 -/- }
\end{aligned}
$$

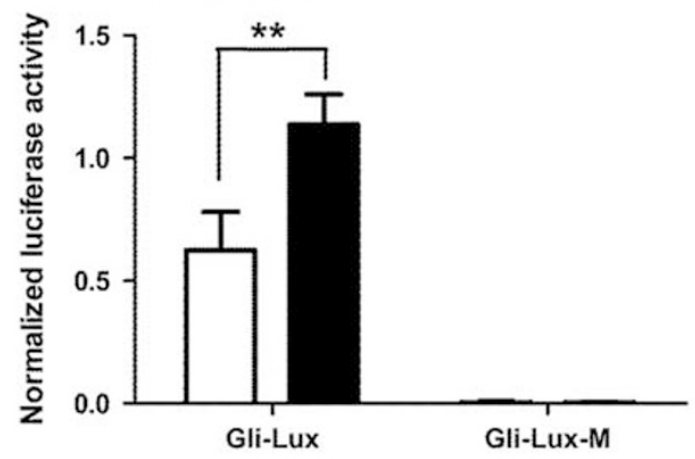

b
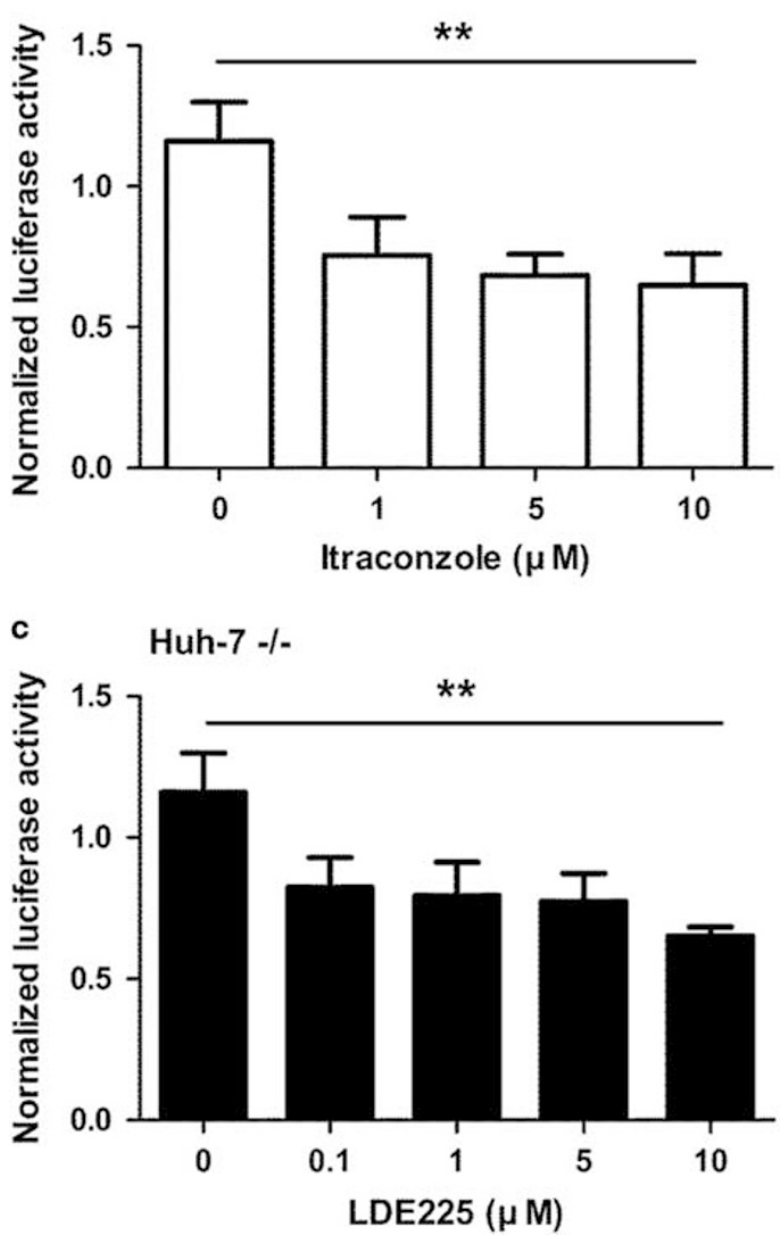

itraconazole, LDE225 and a Raf kinase inhibitor, Sorafenib for $24 \mathrm{~h}$. The cell viability was determined by an MTT reagent. As shown in Figures $4 \mathrm{a}$ and $\mathrm{b}$, treatment with itraconazole at $5-10 \mu \mathrm{M}$ resulted in a decrease in cell viability by 13.15 and $14.57 \%$ in $\mathrm{CD}_{133^{+}} / \mathrm{EpCAM}^{+}$subpopulation, but no difference was observed in the other three subpopulations. Treatment with sorafenib at $10 \mu \mathrm{M}$ significantly compromised

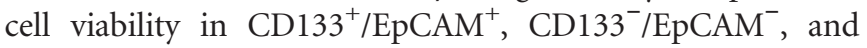

Figure 3 Hedgehog signaling activity as reflected by Gli-Lux reporter

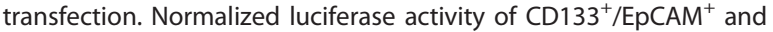

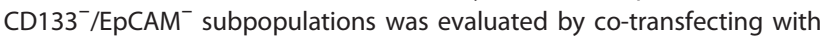
the Gli-Lux in which firefly luciferase is under the control of the Gli promoter and pRL-TK luciferase reporter system in which renilla luciferase is under the control of the TK promoter. (a) Relative luciferase activity in

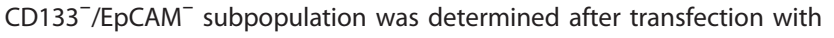
Gli-Lux and a mutated plasmid (Gli-Lux-M). ${ }^{*} P<0.01$ compared with

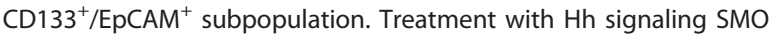
antagonists, itraconazole (b), or LDE225 (c) resulted in a decrease in firefly luciferase activity in $\mathrm{CD} 133^{-} / \mathrm{EpCAM}^{-}$subpopulation. ${ }^{*} \mathrm{P}<0.01$ compared

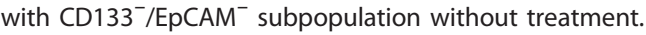

Huh-7-trans subpopulations, but did not affect Huh-7-DN subpopulation (Figures 4c-f). Neither itraconazole, LDE225, nor sorafenib $(10 \mu \mathrm{M})$ suppressed cell viability of Huh-7-DN subpopulation. Moreover, Huh-7-DN and Huh-7-trans subpopulations were treated with various concentrations of sorafenib to determine the effects of hedgehog signaling activity on drug sensitivity. Hep3B cells that are well differentiated in hepatic gene expression profile and exhibit positive CD133/EpCAM expression profile, were used as a control. ${ }^{10}$ The IC50 was calculated to reflect resistance to sorafenib in Hep3B, Huh-7-DN, and Huh-7-trans cell types, and was higher in Huh-7-DN $(21.58 \mu \mathrm{M})$ than $11.6 \mu \mathrm{M}$, and $17.87 \mu \mathrm{M}$ in Hep3B and Huh-7-trans cells (Supplementary Figures 2A-D). Taken together, the cell viability assay documented that Huh-7-DN possessed intrinsic chemoresistance to sorafenib.

\section{Increased Expression of Hh Signaling Molecules Contributed to Chemoresistance of Huh-7-DN Subpopulation}

In order to reveal the controlling mechanisms of chemoresistance of Huh-7-DN subpopulation, expression of $\mathrm{Hh}$ signaling transcription factors $G l i-1 / 2$ and MDR-related $\mathrm{ABC}$ transporters $(A B C B 1, A B C C 1$, and $A B C G 2)$ was determined in various hepatoma subpopulations. Abnormal activation of $\mathrm{Hh}$ signaling in Huh-7-DN and Huh-7-trans subpopulations resulted in an increase in mRNA levels of Gli$1 / 2$ and $\mathrm{Bcl}-2$ genes (Figures $5 \mathrm{a}-\mathrm{c}$ ). Compared with relatively

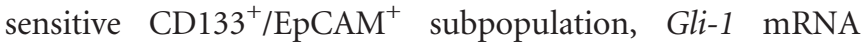
levels of Huh-7-DN and Huh-7-trans subpopulations were increased more than 12- and 22-fold. Gli-2 mRNA levels of Huh-7-DN and Huh-7-trans subpopulations were increased more than 31- and 35-fold. Of note, $A B C C 1$ expression levels were higher in Huh-7-DN and Huh-7-trans cells than the other cell types tested (Figure 5d). Therefore, higher expression of Gli-1 and Gli-2 was accompanied with upregulation of $A B C C 1$ expression in Huh-DN and Huh-7trans subpopulations (2- and 3.5-fold). In contrast, expression levels of $A B C B 1$ and $A B C G 2$ in Huh-7-DN and Huh-7-trans subpopulations were much lower than unsorted Huh-7 and $\mathrm{CD} 33^{+} / \mathrm{EpCAM}^{+}$subpopulations (Figures 5e and f). Although 

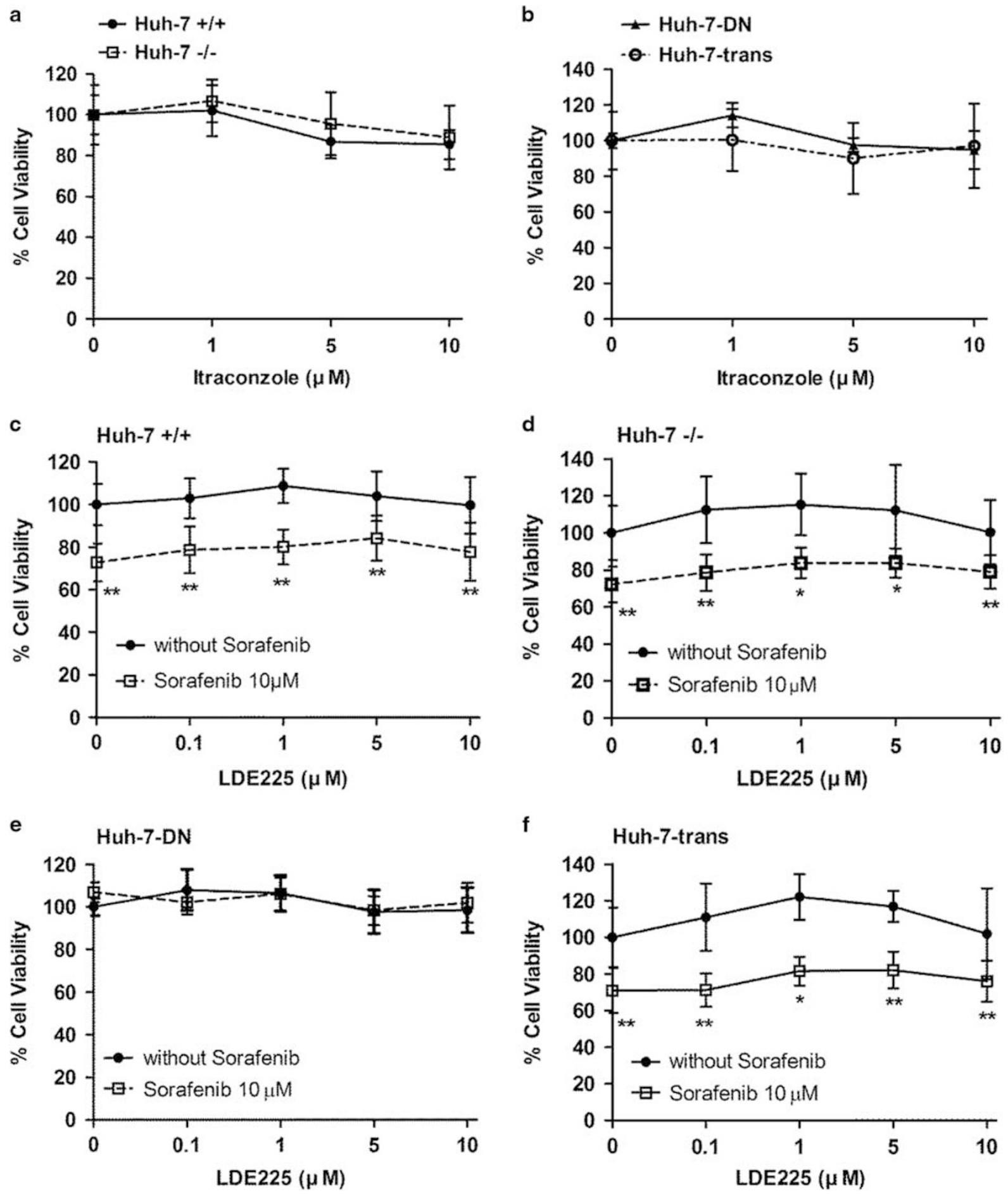

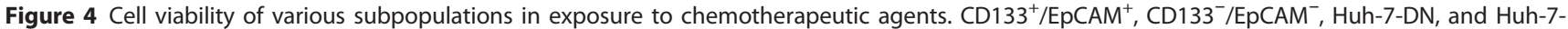
trans subpopulations were treated with chemotherapeutic drugs as indicated concentration for $24 \mathrm{~h}$. Cell viability was determined by MTT and

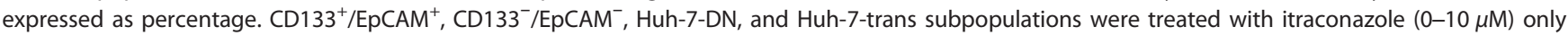
(a and b); or with LDE225 $(0-10 \mu \mathrm{M})$ plus/minus sorafenib at $10 \mu \mathrm{M}(\mathbf{c}-\mathbf{f}) .{ }^{*} P<0.05,{ }^{* *} P<0.01$ compared with LDE225 only at corresponding concentrations.

Gli-1 and Gli-2 mRNA levels were much higher in Huh-7-DN and Huh-7-trans subpopulations than other hepatoma cells, only Gli-2 protein level was strikingly increased in both Huh-7-DN and Huh-7-trans subpopulations (Figure 6a).
Consistent with real-time PCR results, immunofluorescent staining exhibited a similar increase in $\mathrm{ABCC} 1$ protein level in Huh-7-DN subpopulation (Figure 6b). Moreover, the ABCC1 protein level of Huh-7-DN was confirmed to be significantly 
a

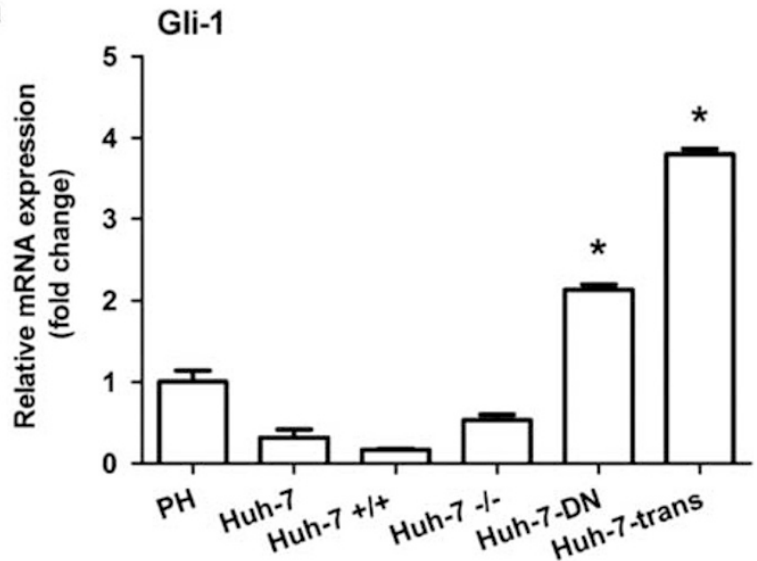

C

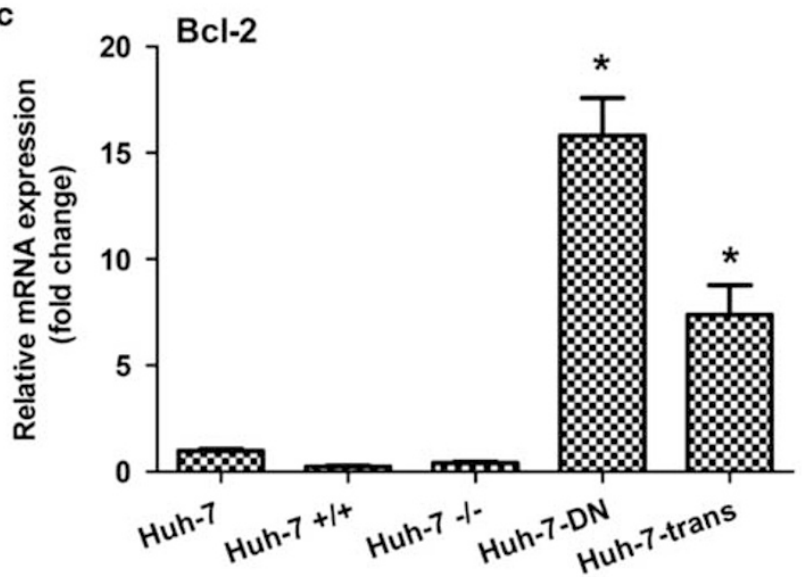

e

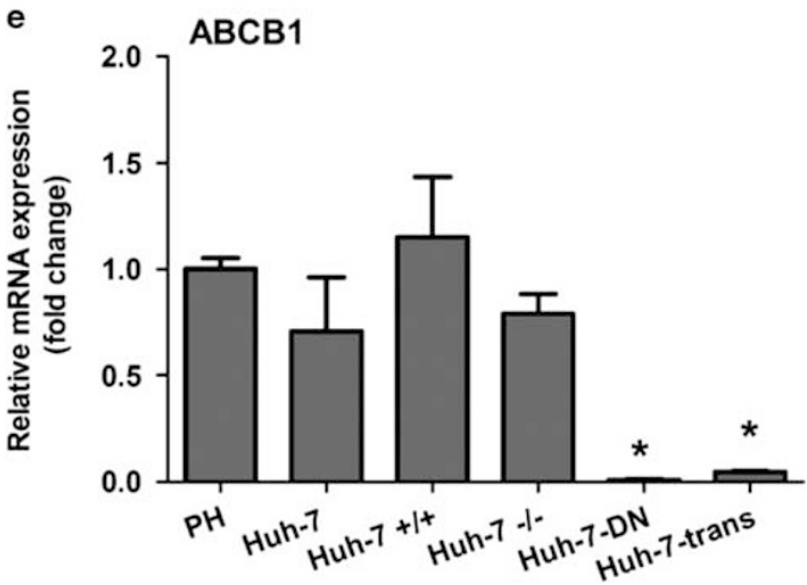

b

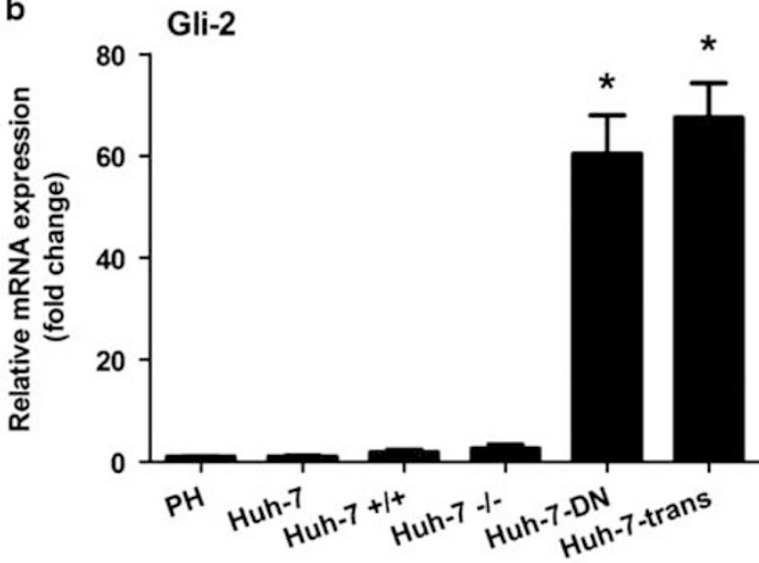

d

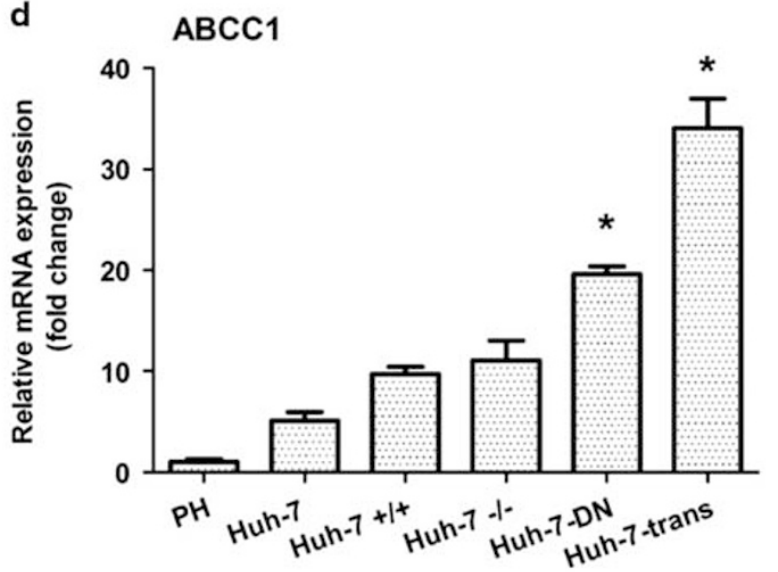

f

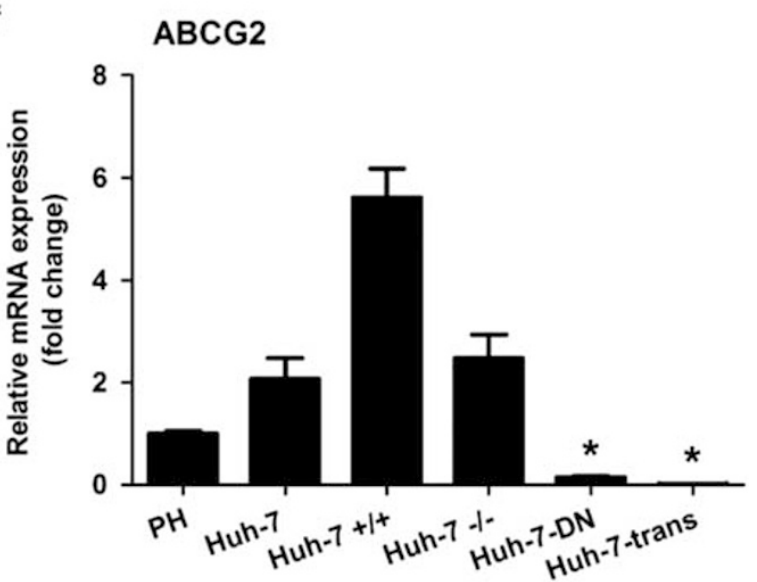

Figure 5 Expression of $\mathrm{Hh}$ signaling pathway and multidrug-resistant $A B C$ transporters in various subpopulations. Expression of $\mathrm{Hh}$ signaling transcription factors, Gli-1 and Gli-2; ABC transporters $A B C C 1, A B C B 1, A B C G 2$ was determined in Huh-7, CD133 ${ }^{+} / \mathrm{EpCAM}^{+}$, CD133 ${ }^{-} / \mathrm{EpCAM}^{-}$, Huh-7-DN, and Huh-7-trans subpopulations by quantitative RT-PCR using Huh-7 as a control. (a and b) Relative Gli-1 and Gli-2 mRNA expression over primary human hepatocyte (PH). (c) Relative $B C L-2$ mRNA expression levels (fold) over Huh-7 cells. (d-f) Relative $A B C C 1, A B C B 1$, and $A B C G 2$ mRNA expression levels (fold) over primary human hepatocyte $(\mathrm{PH}) .{ }^{*} \mathrm{P}<0.05$ compared with Huh-7.

higher than Huh-7-trans subpopulation (Figures 6a and f). These findings indicate that the Hh signaling has a crucial role in the development of chemoresistance in Huh-7-DN subpopulation through the ABCC1 transporter.
Pharmacological Suppression of the Hh Signaling Activity Decreased ABCC1 Expression

To further investigate whether Hh signaling is involved in the regulation of $A B C C 1$ transporter gene expression, Huh-7-DN 
a
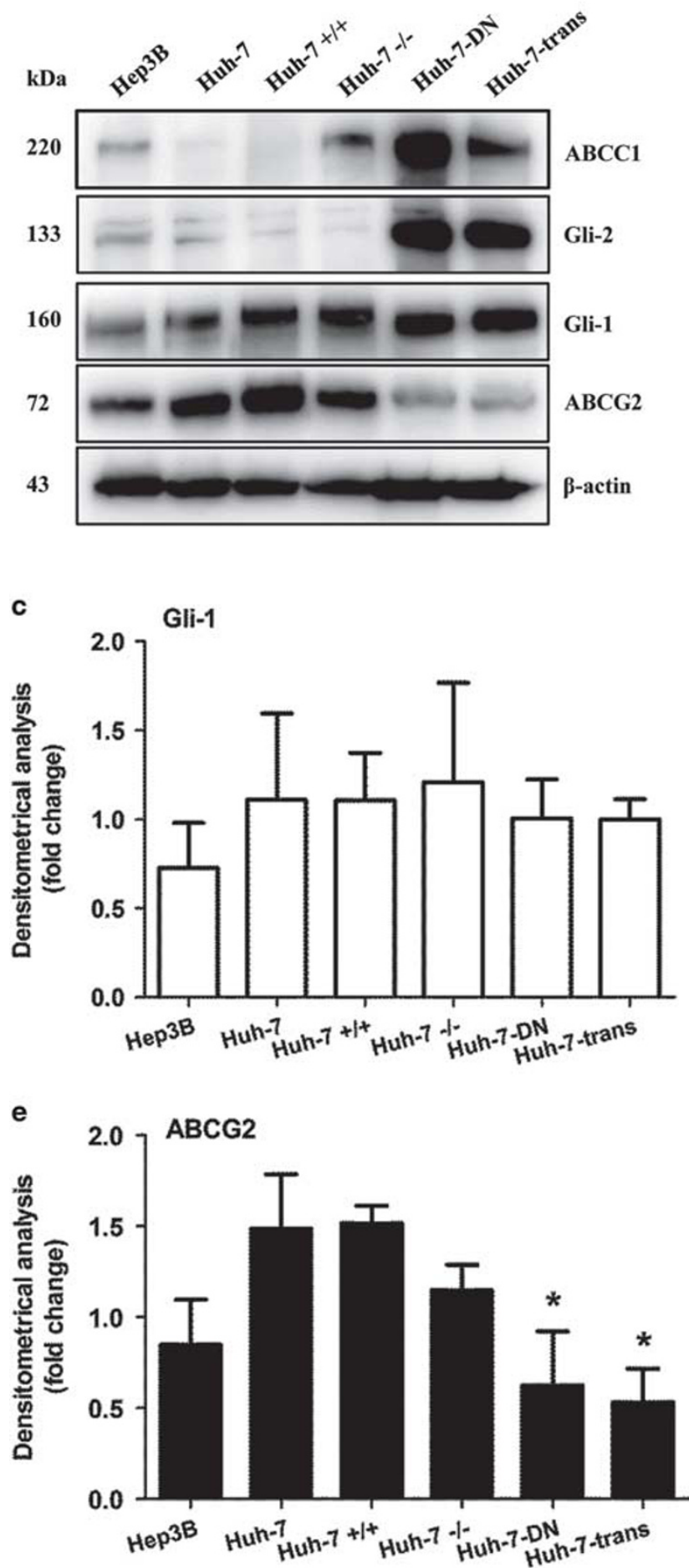
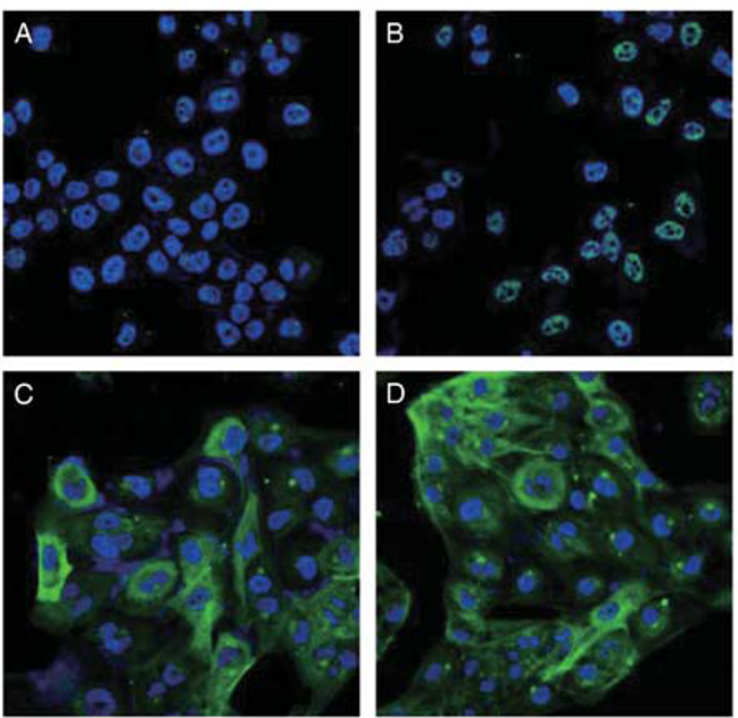

d
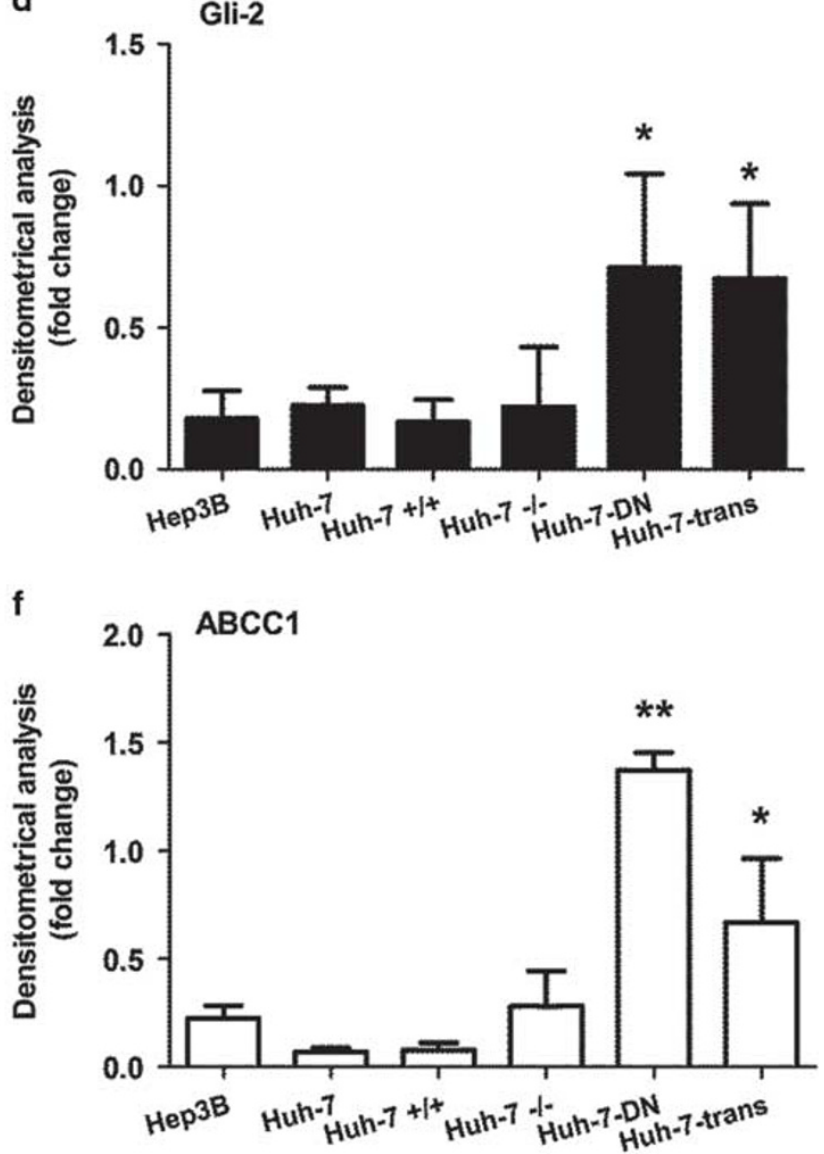

Figure 6 Protein levels of Hh signaling pathway molecules and ABC transporters in various hepatoma cells. (a) Gli-1/2, ABCC1, and ABCG2 protein levels as determined by western blot analysis, and (c-f) arbitrary value of densitometrical analysis of three independent experiments is shown, using $\beta$-actin as a loading control. ${ }^{*} P<0.05,{ }^{*} P<0.01$ compared with Huh-7. (b) Immunofluorescent staining of ABCC1 in CD133 ${ }^{+} / \mathrm{EpCAM}^{+}$(A), CD133 $/ \mathrm{EpCAM}^{-}$(B), Huh7-DN (C), and Huh-7-trans (D). Magnification: $\times 400$.

and Huh-7-trans subpopulations were treated with $\mathrm{Hh}$ signaling SMO antagonists, LDE225 or itraconazole. As shown in Figures 7 and 8, neither itraconazole nor LDE225- affected Gli-1 mRNA levels in all concentrations tested in both Huh-7-DN and Huh-7-trans subpopulations, whereas Gli-2 expression was suppressed by a high concentration of 
LDE225 $(10 \mu \mathrm{M})$ in both Huh-7-DN $(P=0.016)$ and Huh-7trans subpopulations $(P=0.017$; Figures $7 \mathrm{~b}$ and $8 \mathrm{~b})$. A significant inhibitory effect on Gli-2 expression was observed
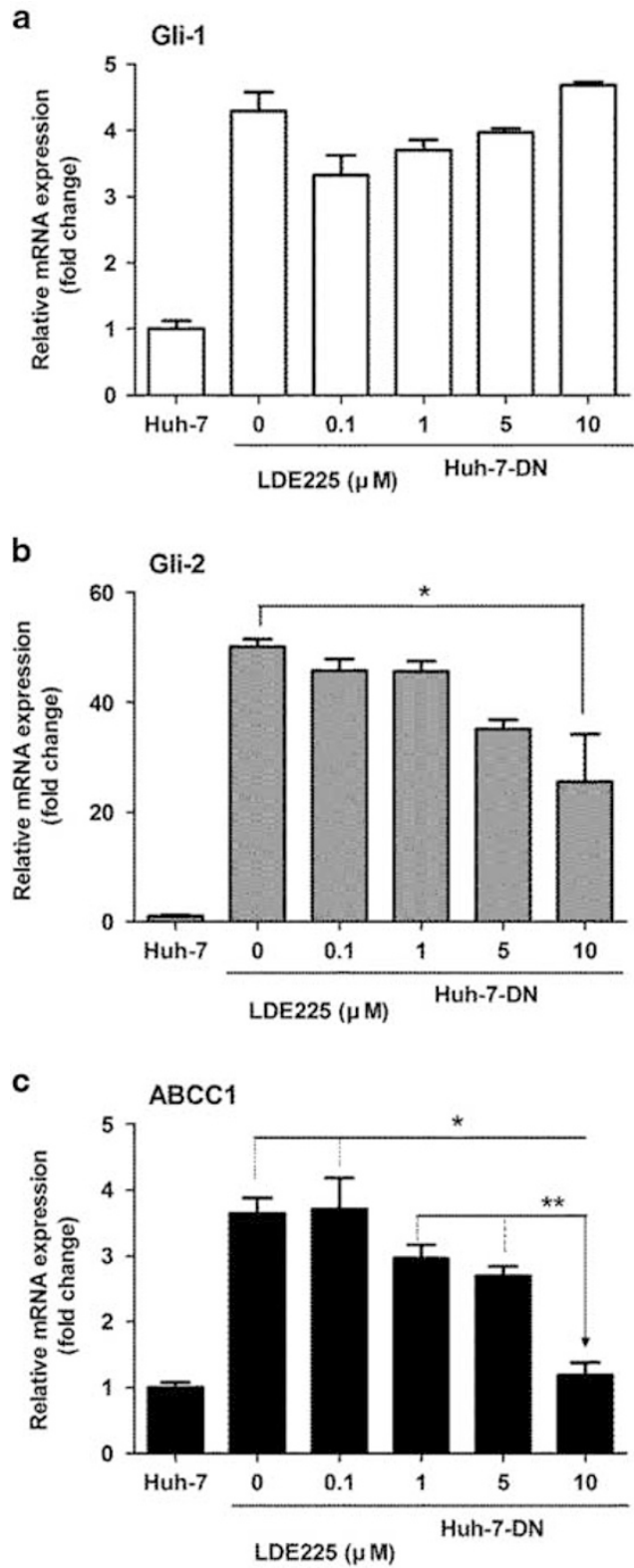

g

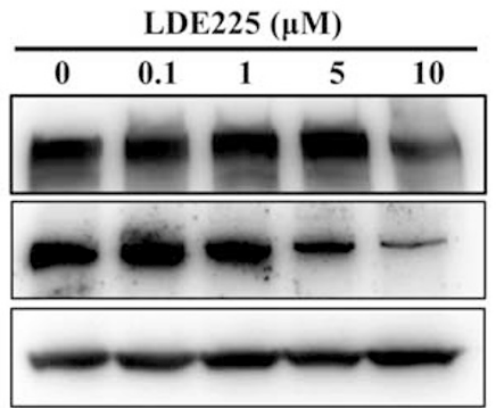

Huh-7-DN by itraconazole at $1 \mu \mathrm{M}$, and a dose-dependent decrease of Gli-2 was seen at high concentrations $(5-10 \mu \mathrm{M})$ in Huh-7trans subpopulation (Figure 8e). Both LDE225 and
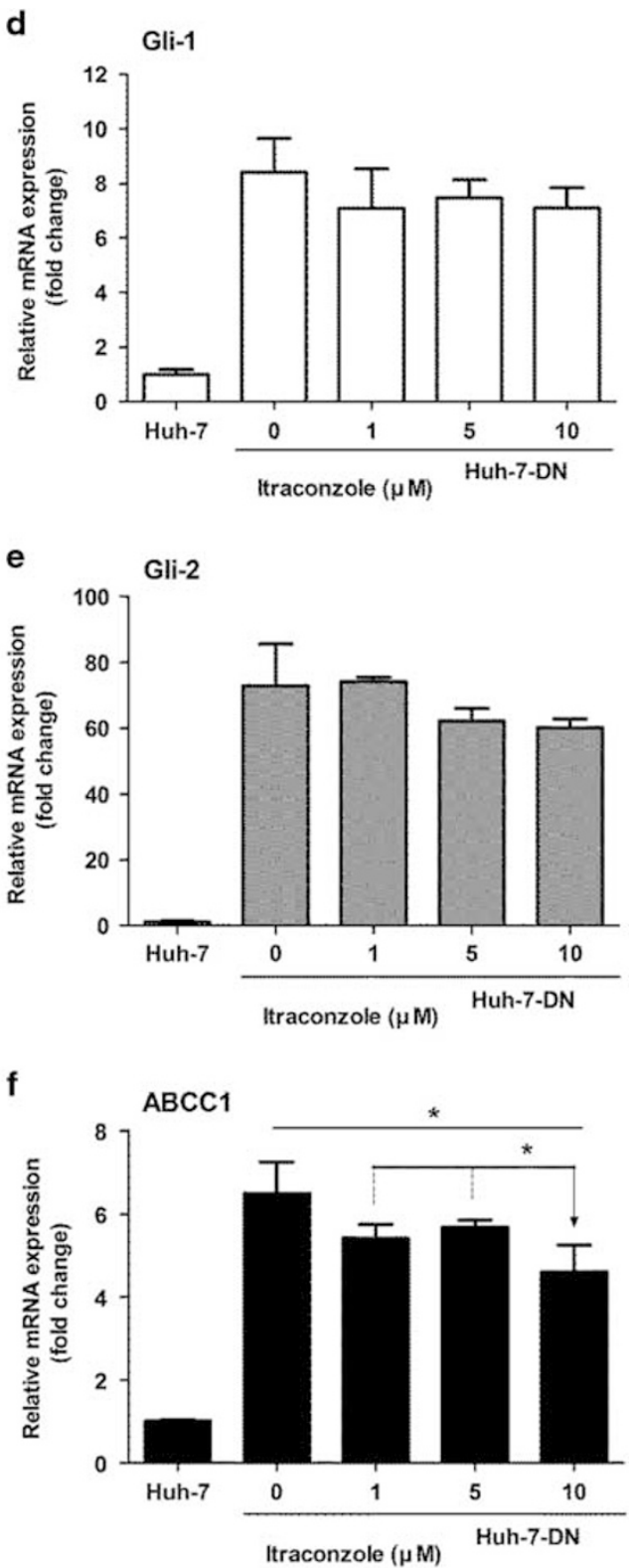

h

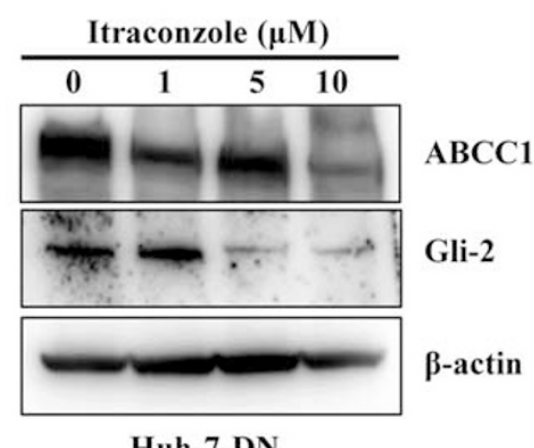

Huh-7-DN 
itraconazole suppressed expression of $A B C C 1$ in Huh-7-DN subpopulation starting at $1 \mu \mathrm{M}$ in a dose-dependent manner, and the most obvious decrease of $A B C C 1$ mRNA expression was observed at $10 \mu \mathrm{M}$ (Figures $7 \mathrm{c}$ and f). Consistent with mRNA levels, ABCC1 protein levels were significantly reduced in Huh-7-DN by LDE225 or itraconazole at $10 \mu \mathrm{M}$, whereas the reduction of Gli-2 protein levels occurred when the concentration of LDE225 and itraconazole was increased to $5 \mu \mathrm{M}$ (Figures $7 \mathrm{~g}$ and $\mathrm{h}$ ). Both LDE225 and itraconazole $(1-10 \mu \mathrm{M})$ significantly reduced $A B C C 1$ mRNA expression in Huh-7-trans subpopulation, and subsequently western blot analysis confirmed that $\mathrm{ABCC} 1$ protein levels decrease from $1.0 \mu \mathrm{M}$ (Figures $8 \mathrm{~g}$ and $\mathrm{h}$ ). These data further verified that $\mathrm{Hh}$ signaling transcription factor Gli-2 appeared to be the primary regulator for drug sensitivity of Huh-7-DN and Huh-7-trans subpopulations through $A B C C 1$ transporter.

\section{DISCUSSION}

In the present study, we compared the phenotypic features of a newly established subpopulation of Huh-7-DN with the previously reported Huh-7-trans subpopulation, ${ }^{14}$ and other subpopulations or hepatoma cells, and characterized their sensitivity to sorafenib, LDE225 and another Hh inhibitor, itraconazole. The latter is an antifungal agent, and was defined as a potent $\mathrm{Hh}$ inhibitor that targets SMO and is distinct from other $\mathrm{Hh}$ inhibitors, such as LDE225 or cyclopamine. $^{23}$ In contrast to the well-differentiated $\mathrm{CD} 133^{+} / \mathrm{EpCAM}^{+}$subpopulation that possessed epithelial morphology and $\alpha$-fetoprotein expression, ${ }^{10} \mathrm{Huh}-7-\mathrm{DN}$ and Huh-7-trans cells derived from the CD133-/EpCAM ${ }^{-}$subpopulation are unique subpopulations characterized by the occurrence of EMT (Figure 2) and enhanced hedgehog signaling activity (Figure 3). These $\mathrm{CD}_{133} / \mathrm{EpCAM}^{-}$subpopulations have been previously demonstrated to be poorly differentiated in terms of their hepatocyte-specific gene expression profile and function. ${ }^{10}$ Not only did both Huh7-DN and Huh-7-trans subpopulations retain $\mathrm{CD}^{-133^{-}}$/ $\mathrm{EpCAM}^{-}$expression profile, but also they appeared to have striking karyotypic alterations and different transcriptome by RNA-Seq (data not shown). Huh-7-trans subpopulation displayed metastatic capability with upregulation of matrix metalloproteinase (MMP)-1/2/9 as we reported recently. ${ }^{14}$ Huh-7-DN subpopulation exhibited much more resistance to sorafenib than Hun-7-trans and other subpopulations. The viability of Huh-7-DN was not compromised by structurally unrelated chemotherapeutic agents in single use or combination (sorafenib, LDE225, and itraconazole; Figure 4); however, both LDE225 and itraconazole suppressed Hh signaling activity in Huh-7 $\mathrm{CD}^{-3} 3^{-} / \mathrm{EpCAM}^{-}$subpopulation in a dose-dependent manner (Figure 3). These data verify that Huh-7-DN cells possess an intrinsic resistance to the chemotherapeutic agents tested, and could be a valuable tool in exploring how Hh signaling modulates drug resistance in HCC.

To explore the underlying mechanisms of chemoresistance in Huh-7-DN cells, we first examined levels of the $A B C$ transporter molecules, and found that $A B C C 1$ expression was significantly increased in both mRNA and protein levels in Huh-7-DN compared with other subpopulations. However, the other two $\mathrm{ABC}$ transporters $(A B C B 1$ and $A B C G 2)$ were decreased in both Huh-7-DN and Huh-7-trans cells compared with other subpopulations. At the same time, Gli-2 expression levels in both Huh-7-DN and Huh-7-trans cells were much higher (61- and 68-fold compared with Huh-7 cells) than other subpopulations, though there was a moderate increase in Gli-1 level in Huh-7 DN (6.8-fold), and a larger increase in Huh-7-trans cells (12.2-fold) compared with Huh-7 cells. The aberrant Gli-1 expression, especially the occurrence of truncated Gli-1, was thought to be responsible for the highly metastatic property in Huh-7trans subpopulation as we previously reported. ${ }^{14}$ Based on these results, it is reasonable to believe that Gli-2 has a more dominant role than Gli-1 in the mediation of chemoresistance in Huh-7-DN subpopulation. Our findings are consistent with a notion that $A B C C 1$ is not expressed in mature hepatocytes (Figure 5d), and its expression levels are negatively correlated with differentiation grade in untreated HCC. ${ }^{24}$ Moreover, constitutive activation of the Hh pathway maintains chemoresistance through increasing drug efflux of $\mathrm{ABC}$ transporters (ABCB1 and $A B C G 2),{ }^{25}$ and Gli-1 maintained multidrug-resistant phenotype of myeloid leukemia by $\mathrm{ABCB} 1{ }^{26}$ These findings are further supported by the observation that $\mathrm{ABCB} 1$ and $\mathrm{ABCG} 2$ promoter regions have the consensus sequence of Gli-binding site, and Gli-1 directly bound to the consensus GACCACCCA-like motif located in the promoter regions of $\mathrm{ABCB} 1$ and $\mathrm{ABCG} 2$ to regulate their transcription in B-cell lymphoma and ovarian cancer cells. $^{27,28}$ However, our results demonstrate that ABCC1 was highly expressed in Huh-7-DN and Huh-7-trans cells; in

Figure 7 The effects of LDE225 and itraconazole on expression of Gli-1, Gli-2, and ABCC1 in Huh-7-DN subpopulation. Huh-7-DN cells were exposed to LDE225 or itraconazole at various concentrations as indicated for $24 \mathrm{~h}$. mRNA levels of Gli-1, Gli-2, and ABCC1 in Huh-7-DN subpopulation were determined by quantitative RT-PCR, using untreated Huh-7 cells as a control. Protein levels of Gli-2 and ABCC1 were determined by western blot analysis, using $\beta$-actin as a loading control. Neither LDE225 nor itraconazole effectively suppressed Gli-1 mRNA expression (a and d), whereas LDE225 $(10 \mu \mathrm{M})$ significantly reduced Gli-2 mRNA expression (b and e). Both LDE225 and itraconazole treatment inhibited ABCC1 mRNA expression in a dosedependent manner, and a maximum inhibitory effect was observed at $10 \mu \mathrm{M}$ (c and f). Gli-2 protein levels started to decrease from 5 to $10 \mu \mathrm{M}$ and ABCC1 protein levels were significantly reduced at $10 \mu \mathrm{M}$ when exposure to LDE225 or itraconazole in Huh-7-DN subpopulation (g and $\mathbf{h})$. ${ }^{*} P<0.05$, ** $P<0.01$ compared with untreated Huh-7-DN subpopulation. 
contrast, $\mathrm{ABCB} 1$ and $\mathrm{ABCG} 2$ were downregulated in these two subpopulations. Accordingly, the protein levels of Gli-2 were remarkably increased in Huh-7-DN and Huh-7-trans
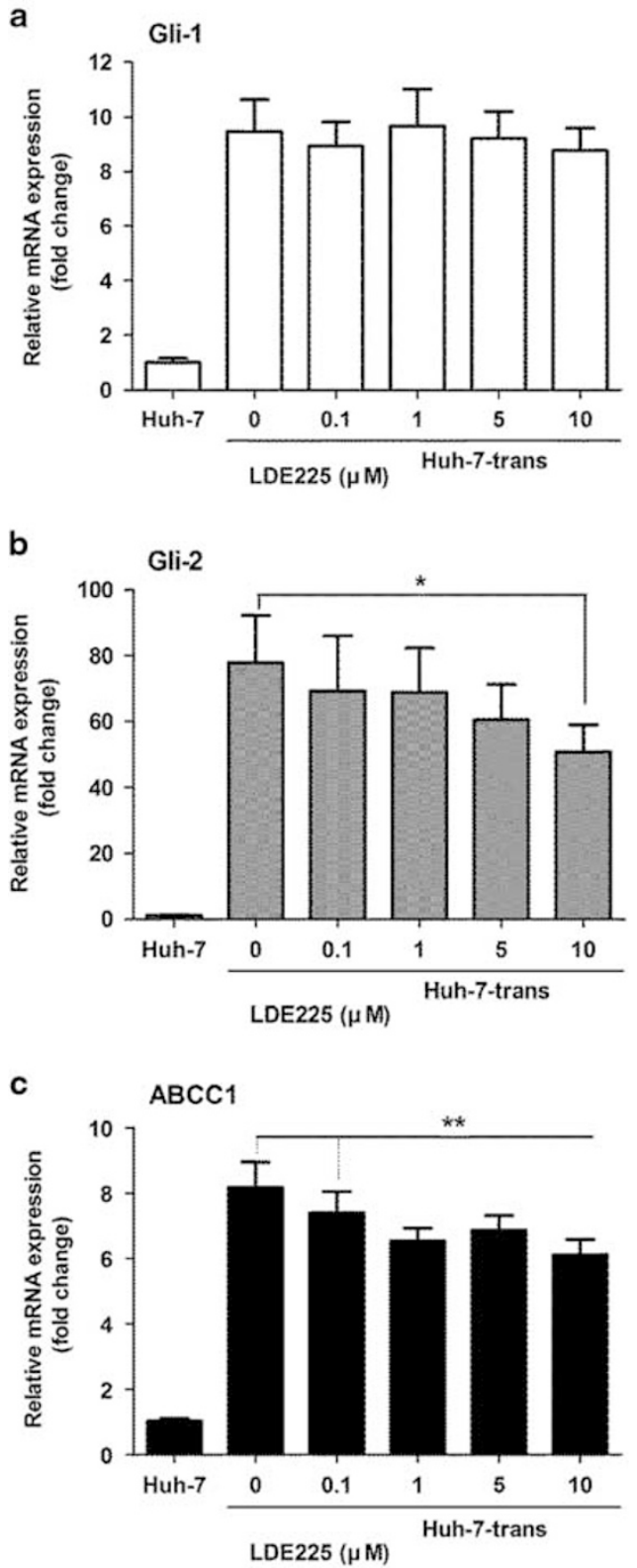

g

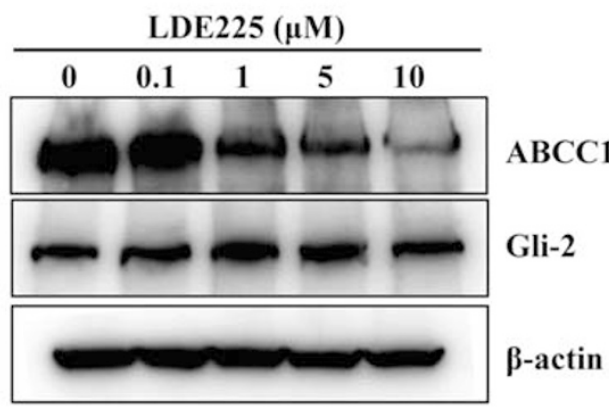

Huh-7-trans subpopulations. Given the fact that both Gli-1 and Gli-2 may bind to the same motif in Gli-binding site and initiate transcription of target genes, we speculate that Gli-2, rather
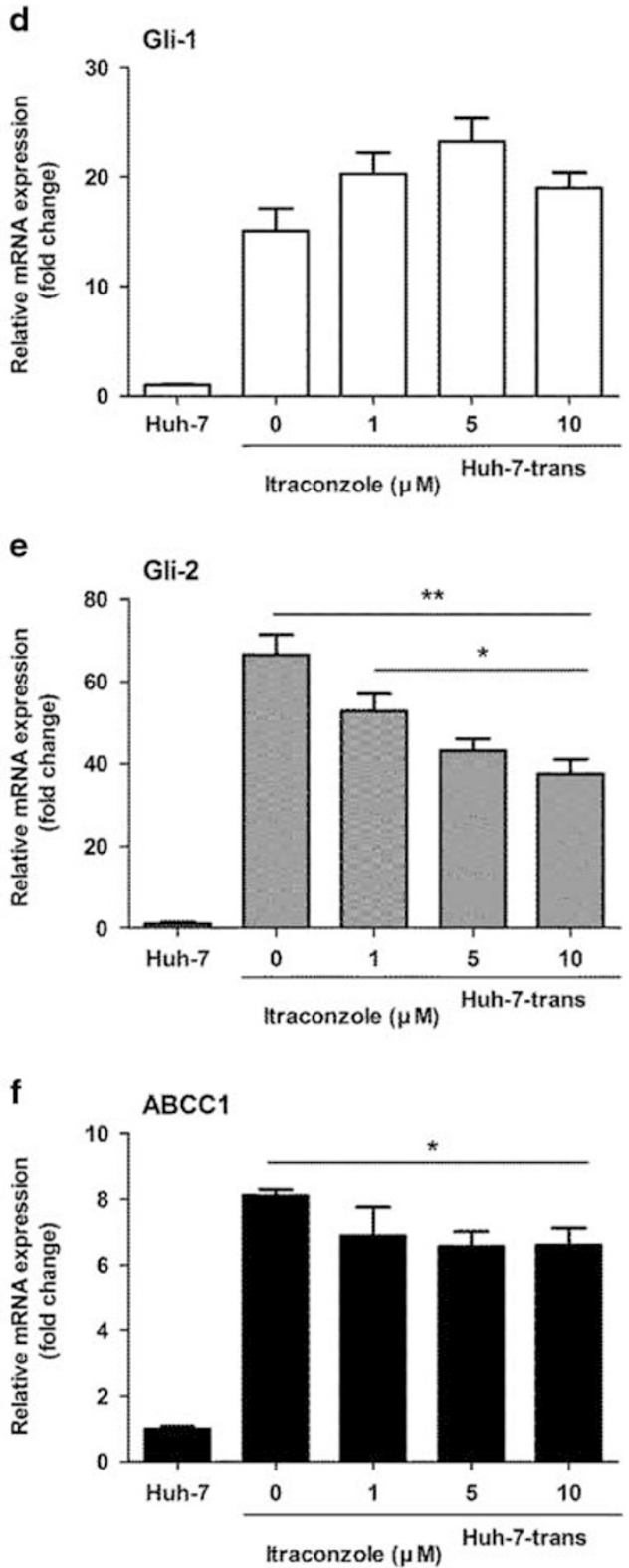

h

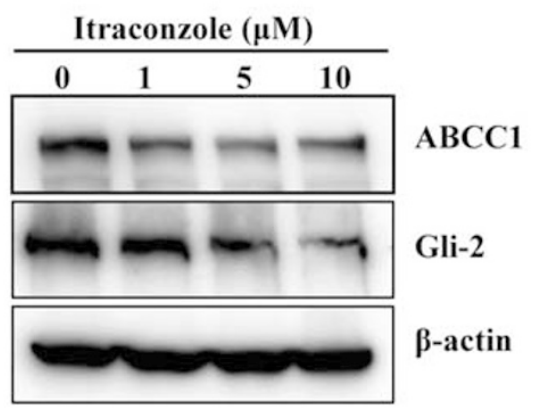

Huh-7-trans 
than Gli-1, is the major transcription factor controlling the resistance of Huh-7-DN subpopulation to sorafenib through the $\mathrm{ABCC} 1$ transporter, which is obviously different from what was observed in myeloid leukemia.

To further confirm the regulation of Gli-2 on $A B C C 1$ expression, itraconazole and LDE225 were separately used to treat various subpopulations. As shown in Figures $7 \mathrm{a}$ and $\mathrm{d}$ and $8 \mathrm{a}$ and d, both LDE225 and itraconazole did not display any inhibition on Gli-1 gene expression in either Huh-7-trans or Huh-7-DN subpopulations. The results demonstrated that inhibition of Gli-2 expression by either itraconazole or LDE225 simultaneously suppressed $A B C C 1$ expression in Huh-7-DN or Huh-7-trans subpopulations (Figures 7 and 8), although the inhibition on Gli-2 expression took place when the concentration of LDE225 was reached to $10 \mu \mathrm{M}$. The discrepancy in suppressing Gli-1 and Gli-2 gene expression by SMO antagonists, LDE225 or itraconazole, may be partially attributed to the fact that hedgehog signaling activation is under the control of canonical and noncanonical pathway, and in noncanonical pathway, hedgehog activation is operated in SMO-independent manner. ${ }^{29}$ This is intriguing, and more experimentation is needed to confirm our speculation. It is also possible that LDE225 was less sensitive in indirectly suppressing Gli expression when compared with its direct inhibition on $\mathrm{Hh}$ signaling activity in $\mathrm{CD}_{133^{-}}$/ EpCAM $^{-}$Huh-7 cells. Drug resistance has apparently developed in these cells because LDE225 has been shown to be effective at $\mathrm{nM}$ levels. ${ }^{14,30}$

To further verify the effect of Gli-2 on $A B C C 1$ expression, we used RNA interference (RNAi) and suppressed Gli-2 expression with transduction of a lentiviral-particle-harboring shRNA against Gli-2 gene in both Huh-7-DN and Huh-7trans cells, and used cells transduced with lentiviral particles harboring scrambled shRNA as a control (Supplementary Figure 3). Our data demonstrate that shRNA against Gli-2 reduced Gli-2 gene expression by $50-70 \%$ at mRNA levels in two clones, and nearly $25-30 \%$ reduction at protein levels in both Huh-7-trans and Huh-7-DN cells $(P<0.05-0.01)$. Subsequently, a $10-20 \%$ decrease $(P<0.05)$ in ABCC1 mRNA and protein levels was seen in cells transduced with a lentiviral particles harboring shRNA against Gli-2 compared with those transduced with a lentiviral vector harboring scrambled shRNA. Therefore, these data partially support that $A B C C 1$ is the target gene of the Hh signaling in both Huh-7DN and Huh-7-trans cells, and Gli-2 has a major role in the development of drug resistance through modulating $A B C C 1$ gene expression, although other factors, such as EMT transcription factors, may also participate in this process as discussed below.

Hh signaling has a critical role during embryonic development, and is often silent in adult tissue. It is activated to participate in tissue repair in chronic damage. ${ }^{31}$ With $\mathrm{Hh}$ pathway activation, hedgehog ligands (Sonic, Indian or Desert) bind to the PTCH1 receptor to release the G-protein-coupled receptor (GPCR)-family protein smoothened (SMO), and the latter leads to stabilization and nuclear translocation of GLI family members. Gli-1/2 then transact target genes, such as B-cell lymphoma 2 (Bcl-2), twist, snail, Gli-1 and PTCH-1..$^{32} \mathrm{SMO}$, one of critical molecules in the Hh signaling pathway, has been a major target for pharmaceutical intervention in cancer therapy, and has been clinically proved to be effective in basal cell cancer and other malignancies although drug resistance has been reported in basal cell cancer against vismodegib. ${ }^{15,33}$ As one of Gli-1/2 target genes, $B C L-2$ exerts its anti-apoptosis effects by binding to pro-apoptotic proteins and preventing the release of cytochrome $c$ from mitochondria. ${ }^{34}$ Consistent with the expression trend of ABCC1 protein, Huh-7-DN subpopulation had the highest $B C L-2$ expression level, which was followed by Huh-7-trans subpopulation. Thus, Hh signaling maintains chemoresistance of hepatoma cells through increasing drug efflux mediated by $A B C C 1$ transporter and promoting cell survival by overexpression of $B c l-2$.

Huh-7-DN and Huh-7-trans cells exhibited EMT features by overexpression of relevant transcription factors, twist and snail. The occurrence of EMT has been implicated as another mechanism of hedgehog signaling in the mediation of MDR (Supplementary Figure 1). Hh pathway activation directly contributes to the expression of twist and snail. ${ }^{35}$ The expression levels of twist in Huh-7-DN and Huh-7-trans cells were 300-400-fold higher than in Huh-7 cells, which indicates that this transcription factor may participate in modulating drug resistance through regulating the EMT status. Hence, it was postulated that aberrant hedgehog signaling activation promotes the transacting activity of twist and snail, which may in turn increase the activity of $\mathrm{ABC}$ transporters in the downstream. It remains to be seen whether Gli-2 directly transacts $A B C C 1$ or indirectly transacts it through activation of twist and/or snail at a transcriptional level in Huh-7-DN cells.

In conclusion, the present study established a poorly

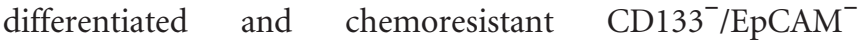

Figure 8 The effects of LDE225 and itraconazole on expression of Gli-1, Gli-2, and ABCC1 in Huh-7-trans subpopulation. Huh-7-trans cells were exposed to LDE225 or itraconazole at various concentrations as indicated for 24 h. mRNA levels of Gli-1, Gli-2, and ABCC1 in Huh-7-trans subpopulation were determined by quantitative RT-PCR, using untreated Huh-7 as a control. Protein levels of Gli-2 and ABCC1 were determined by western blot analysis, using $\beta$-actin as a loading control. Similar to Huh-7-DN subpopulation, LDE225 or itraconazole did not reduce Gli-1 mRNA expression (a and d). LDE225 or itraconazole could not only significantly inhibit Gli-2 mRNA, but also ABCC1 mRNA expression (b, $\mathbf{c}$, e and f). ABCC1 protein levels started to decrease when LDE225 or itraconazole concentration reached to $1.0 \mu \mathrm{M}$ (g and $\mathbf{h}$ ). ${ }^{*} P<0.05,{ }^{*} P<0.01$ compared to untreated Huh-7-trans subpopulation. 
Huh-7-DN subpopulation exhibiting EMT and upregulation of the $A B C C 1$ transporter. These characteristics are attributed to increased expression of $\mathrm{Hh}$ transcription factor Gli-2, which may govern expression of the $A B C C 1$ transporter and contribute to sorafenib resistance of hepatoma cells. Our results reveal the molecular mechanism underlying HCC chemoresistance, and may aid in improving the outcome of chemotherapy in HCC.

Supplementary Information accompanies the paper on the Laboratory Investigation website (http://www.laboratoryinvestigation.org)

\section{ACKNOWLEDGMENTS}

The authors are grateful to Dr Ping Zhou, Stem Cell Program, UC Davis Medical Center, Sacramento, CA, USA for providing CDNA of human primary hepatocytes; and to Mrs Ke Qiao, Shuhui Sun, and Jing Qian in the Departments of Medical Microbiology and Immunology, School of Basic Medical Sciences, Fudan University, for their technical support in the use of confocal microscope and FACS enrichment. The authors thank Professor Zhaoyuan Hou and Dr Yimin Chu at the Faculty of Basic Medicine, Shanghai Jiaotong University School of Medicine, for providing Gli-2 shRNA lentiviral plasmid and their helpful suggestions for Gli-2 knockdown experiments. This work is supported by the National Natural Science Foundation (NSFC \#81272436 and 81572356) and the Ministry of Science and Technology (\#2016YFE0107400), China, to JW as well as the Young Investigator Program of Jing'an District Municipal Commission of Health and Family Planning (JWRC2014Q01) to JD.

\section{DISCLOSURE/CONFLICT OF INTEREST}

The authors declare no conflict of interest.

1. Lozano R, Naghavi M, Foreman $\mathrm{K}$, et al. Global and regional mortality from 235 causes of death for 20 age groups in 1990 and 2010: a systematic analysis for the Global Burden of Disease Study 2010. Lancet 2012;380:2095-2128.

2. Alves RC, Alves D, Guz B, et al. Advanced hepatocellular carcinoma. Review of targeted molecular drugs. Ann Hepatol 2011;10:21-27.

3. Bruix J, Takayama T, Mazzaferro V, et al. Adjuvant sorafenib for hepatocellular carcinoma after resection or ablation (STORM): a phase 3, randomised, double-blind, placebo-controlled trial. Lancet Oncol 2015;16:1344-1354.

4. Samuel M, Chow PK, Chan Shih-Yen E, et al. Neoadjuvant and adjuvant therapy for surgical resection of hepatocellular carcinoma. Cochrane Database Syst Rev 2009; 1: CD001199.

5. Ge S, Huang D. Systemic therapies for hepatocellular carcinoma. Drug Discov Ther 2015:9:352-362.

6. Llovet JM, Ricci S, Mazzaferro V, et al. Sorafenib in advanced hepatocellular carcinoma. N Engl J Med 2008;359:378-390.

7. Kelley RK. Adjuvant sorafenib for liver cancer: wrong stage, wrong dose. Lancet Oncol 2015;16:1279-1281.

8. Tovar V, Cornella H, Moeini A, et al. Tumour initiating cells and IGF/FGF signalling contribute to sorafenib resistance in hepatocellular carcinoma. Gut 2017:66:530-540.

9. Sharom FJ. ABC multidrug transporters: structure, function and role in chemoresistance. Pharmacogenomics 2008:9:105-127.

10. Chen $X$, Lingala $S$, Khoobyari $S$, et al. Epithelial mesenchymal transition and hedgehog signaling activation are associated with chemoresistance and invasion of hepatoma subpopulations. J Hepatol 2011;55: 838-845.

11. Chen XL, Cheng QY, She MR, et al. Expression of sonic hedgehog signaling components in hepatocellular carcinoma and cyclopamineinduced apoptosis through $\mathrm{Bcl}-2$ downregulation in vitro. Arch Med Res 2010;41:315-323.

12. Huang $S$, He J, Zhang $X$, et al. Activation of the hedgehog pathway in human hepatocellular carcinomas. Carcinogenesis 2006;27:1334-1340.
13. Sicklick JK, Li YX, Jayaraman A, et al. Dysregulation of the Hedgehog pathway in human hepatocarcinogenesis. Carcinogenesis 2006;27: 748-757.

14. Fan $\mathrm{YH}$, Ding J, Nguyen $\mathrm{S}$, et al. Aberrant hedgehog signaling is responsible for the highly invasive behavior of a subpopulation of hepatoma cells. Oncogene 2016;35:116-124.

15. Jacobsen $A A$, Aldahan $A S$, Hughes $O B$, et al. Hedgehog pathway inhibitor therapy for locally advanced and metastatic basal cell carcinoma: a systematic review and pooled analysis of interventional studies. JAMA Dermatol 2016:152:816-824.

16. Burness CB. Sonidegib: first global approval. Drugs 2015;75:1559-1566.

17. Lingala S, Cui YY, Chen X, et al. Immunohistochemical staining of cancer stem cell markers in hepatocellular carcinoma. Exp Mol Pathol 2010;89:27-35.

18. Sasaki $K$, Matsuda $M$, Ohkura $Y$, et al. In hepatocellular carcinomas, any proportion of poorly differentiated components is associated with poor prognosis after hepatectomy. World J Surg 2014;38: 1147-1153.

19. Liu L, Zern MA, Lizarzaburu ME, et al. Poly(cationic lipid)-mediated in vivo gene delivery to mouse liver. Gene Ther 2003;10:180-187.

20. Zhang Y, Gu J, Zhao L, et al. Complete elimination of colorectal tumor xenograft by combined manganese superoxide dismutase with tumor necrosis factor-related apoptosis-inducing ligand gene virotherapy. Cancer Res 2006;66:4291-4298.

21. Wege H, Le HT, Chui MS, et al. Telomerase reconstitution immortalizes human fetal hepatocytes without disrupting their differentiation potential. Gastroenterology 2003;124:432-444.

22. Wu J, Liu L, Yen RD, et al. Liposome-mediated extracellular superoxide dismutase gene delivery protects against acute liver injury in mice. Hepatology 2004:40:195-204.

23. Kim J, Tang JY, Gong R, et al. Itraconazole, a commonly used antifungal that inhibits Hedgehog pathway activity and cancer growth. Cancer Cell 2010;17:388-399.

24. Vander Borght $\mathrm{S}$, Komuta $\mathrm{M}$, Libbrecht $\mathrm{L}$, et al. Expression of multidrug resistance-associated protein 1 in hepatocellular carcinoma is associated with a more aggressive tumour phenotype and may reflect a progenitor cell origin. Liver Int 2008;28:1370-1380.

25. Sims-Mourtada J, Izzo JG, Ajani J, et al. Sonic Hedgehog promotes multiple drug resistance by regulation of drug transport. Oncogene 2007;26:5674-5679.

26. Queiroz KC, Ruela-de-Sousa RR, Fuhler GM, et al. Hedgehog signaling maintains chemoresistance in myeloid leukemic cells. Oncogene 2010;29:6314-6322.

27. Singh RR, Kunkalla $\mathrm{K}, \mathrm{Qu} \mathrm{C}$, et al. ABCG2 is a direct transcriptional target of hedgehog signaling and involved in stroma-induced drug tolerance in diffuse large B-cell lymphoma. Oncogene 2011;30: 4874-4886.

28. Chen $Y$, Bieber MM, Teng NN. Hedgehog signaling regulates drug sensitivity by targeting $A B C$ transporters $A B C B 1$ and $A B C G 2$ in epithelial ovarian cancer. Mol Carcinog 2014:53:625-634.

29. Blotta S, Jakubikova J, Calimeri T, et al. Canonical and noncanonical Hedgehog pathway in the pathogenesis of multiple myeloma. Blood 2012;120:5002-5013.

30. Fendrich $\mathrm{V}$, Wiese $\mathrm{D}$, Waldmann J, et al. Hedgehog inhibition with the orally bioavailable Smo antagonist LDE225 represses tumor growth and prolongs survival in a transgenic mouse model of islet cell neoplasms. Ann Surg 2011;254:818-823.

31. Omenetti A, Choi S, Michelotti G, et al. Hedgehog signaling in the liver. J Hepatol 2011;54:366-373.

32. Scales SJ, de Sauvage FJ. Mechanisms of Hedgehog pathway activation in cancer and implications for therapy. Trends Pharmacol Sci 2009;30: 303-312.

33. Gonnissen A, Isebaert S, Haustermans K. Targeting the Hedgehog signaling pathway in cancer: beyond Smoothened. Oncotarget 2015;6: 13899-13913.

34. Kim R, Emi M, Tanabe K. Role of mitochondria as the gardens of cell death. Cancer Chemother Pharmacol 2006;57:545-553.

35. Kong Y, Peng Y, Liu Y, et al. Twist1 and Snail link Hedgehog signaling to tumor-initiating cell-like properties and acquired chemoresistance independently of $A B C$ transporters. Stem Cells 2015;33: 1063-1074. 